\title{
Femtosecond real-time probing of reactions. IV. The reactions of alkali halides
}

\author{
Todd S. Rose, a) Mark J. Rosker, and Ahmed H. Zewail \\ Arthur Amos Noyes Laboratory of Chemical Physics, California Institute of Technology, Pasadena, \\ California 91125
}

(Received 30 June 1989; accepted 15 August 1989)

The photodissociation dynamics of some alkali halides are explored via the method of femtosecond transition-state spectroscopy (FTS). The alkali halide dissociation reaction is influenced by the interaction between the covalent and the ground state ionic potential energy surfaces (PES), which cross at a certain internuclear separation. Depending upon the adiabaticity of the PES, the dissociating fragments may be trapped in a well formed by the avoided crossing of these surfaces. Here, we detail the FTS results of this class of reactions, with particular focus on the reaction of sodium iodide: $\mathrm{NaI}^{*} \rightarrow[\mathrm{Na}---\mathrm{I}]^{+*} \rightarrow \mathrm{Na}+\mathrm{I}$. As in our first report [T. S. Rose, M. J. Rosker, and A. H. Zewail, J. Chem. Phys. 88, 6672 (1988) ], we observe the dynamical motion of the wave packet along the reaction coordinate and the crossing between the covalent and ionic surfaces. The studies presented here characterize the effects of various experimental parameters, including pump and probe wavelengths, on the dynamics of the dissociation and its detection. Comparisons of the results with classical and quantum mechanical calculations are also presented.

\section{INTRODUCTION}

The observation and analysis of chemical bond dissociation (formation) on the femtosecond and picosecond time scale have been presented in previous publications. ${ }^{1-6}$ In these articles, ${ }^{1-6}$ femtosecond transition-state spectroscopy (FTS $)^{7}$ has been developed as a probing method and has been applied to several reactions. This paper, the fourth of the series, ${ }^{1}$ focuses upon the reactions of the alkali halides, particularly that of NaI. Preliminary results for this reaction were described in earlier communications. ${ }^{8,9}$ Here, new FTS measurements, which allow for a more detailed analysis of the dynamics, are given.

In FTS, an initial pump pulse excites the molecule to a dissociative surface (see Fig. 1). A time-delayed tunable probe pulse then monitors the evolution of the molecule on that surface either by tracking the appearance of free fragments (on-resonance detection) or quasi-bound transitionstate species (off-resonance detection). The shapes of the transients, i.e., the time-dependent absorption, can be used to deduce the dynamics of the reaction. ${ }^{1}$ In the case of the ICN reaction,

$$
\mathrm{ICN}^{*} \rightarrow[\mathrm{I}--\mathrm{CN}]^{+*} \rightarrow \mathrm{I}\left({ }^{2} P_{3 / 2},{ }^{2} P_{1 / 2}\right)+\mathrm{CN}\left(X^{2} \Sigma^{+}\right),
$$

the separation of the excited species into two ground state fragments occurs via a "purely" dissociative surface $\left(V_{1}\right.$ in Fig. 1), as discussed in Refs. 1(a) and 1(b). In contrast to the dissociation reaction of $\mathrm{ICN}$, the reaction of $\mathrm{HgI}_{2}$ involves a multidimensional energy surface possessing a saddle point. Motion perpendicular to the dissociation coordinate results in vibrational excitation of the $\mathbf{H g I}$ fragment. This effect was recently observed, ${ }^{6}$ and will be discussed in the following paper, ${ }^{10}$ number $\mathrm{V}$ in the series.

\footnotetext{
a) Present address: The Aerospace Corporation, Department of Lasers and Optics, P.O. Box 92957, MS M2/246, Los Angeles, CA 90009.
}

In contrast to purely dissociative reactions, the reaction of the alkali halides

$$
\mathrm{MX}^{*} \rightarrow[\mathrm{M}--\mathrm{X}]^{+*} \rightarrow \mathrm{M}\left({ }^{2} S_{1 / 2}\right)+\mathrm{X}\left({ }^{2} P_{3 / 2},{ }^{2} P_{1 / 2}\right)
$$

(where $M$ represents the alkali metal and $X$ represents the halogen) is affected by the crossing of the ionic ground state with the lowest excited covalent state(s) (see Fig. 2). It is this characteristic of the alkali halides that leads to interesting effects in the dynamics and has drawn the attention of many researchers since the early days of crossed molecular beam experiments. ${ }^{11}$ Berry, in a classic series of papers, has addressed the nature of the potential energy surfaces for these systems. ${ }^{12}$ The asymptotic atomic states $\mathrm{M}\left({ }^{2} S_{1 / 2}\right)$ and $\mathrm{X}\left({ }^{2} P_{3 / 2}\right)$ correlate with the lowest covalent $\mathrm{MX}$ states with the quantum numbers $\Omega=0^{+}$and 1 .

The crossing (or the avoided crossing) of the alkali halide potential surfaces arises from the fact that the energy required to separate the species into ions $\left(\mathbf{M}^{+}+\mathbf{X}^{-}\right)$is larger than the energy necessary for separation into neutral fragments $(M+X)$. Consequently, the ionic ground state asymptotically approaches a higher energy level at large internuclear separation than do the lowest excited covalent states (Fig. 2). The separation between these two asymptotic values is given by the difference between the ionization potential (IP) of the alkali atom and the electron affinity (EA) of the halogen atom. When the crossing radius $R_{x}$ is large enough, the Coulombic interaction is dominant, and $R_{x}$ is given by

$$
e^{2} / R_{x}=\operatorname{IP}(\mathrm{M})-\mathrm{EA}(\mathrm{X}) .
$$

In the case where the interaction between the covalent and ionic surfaces (or, more accurately, curves) is sufficiently weak, the dissociating molecule behaves as if the curves essentially cross. The dissociation path of such a molecule is effectively confined to the initial potential energy surface upon which it was prepared. This diabatic limit is equivalent 


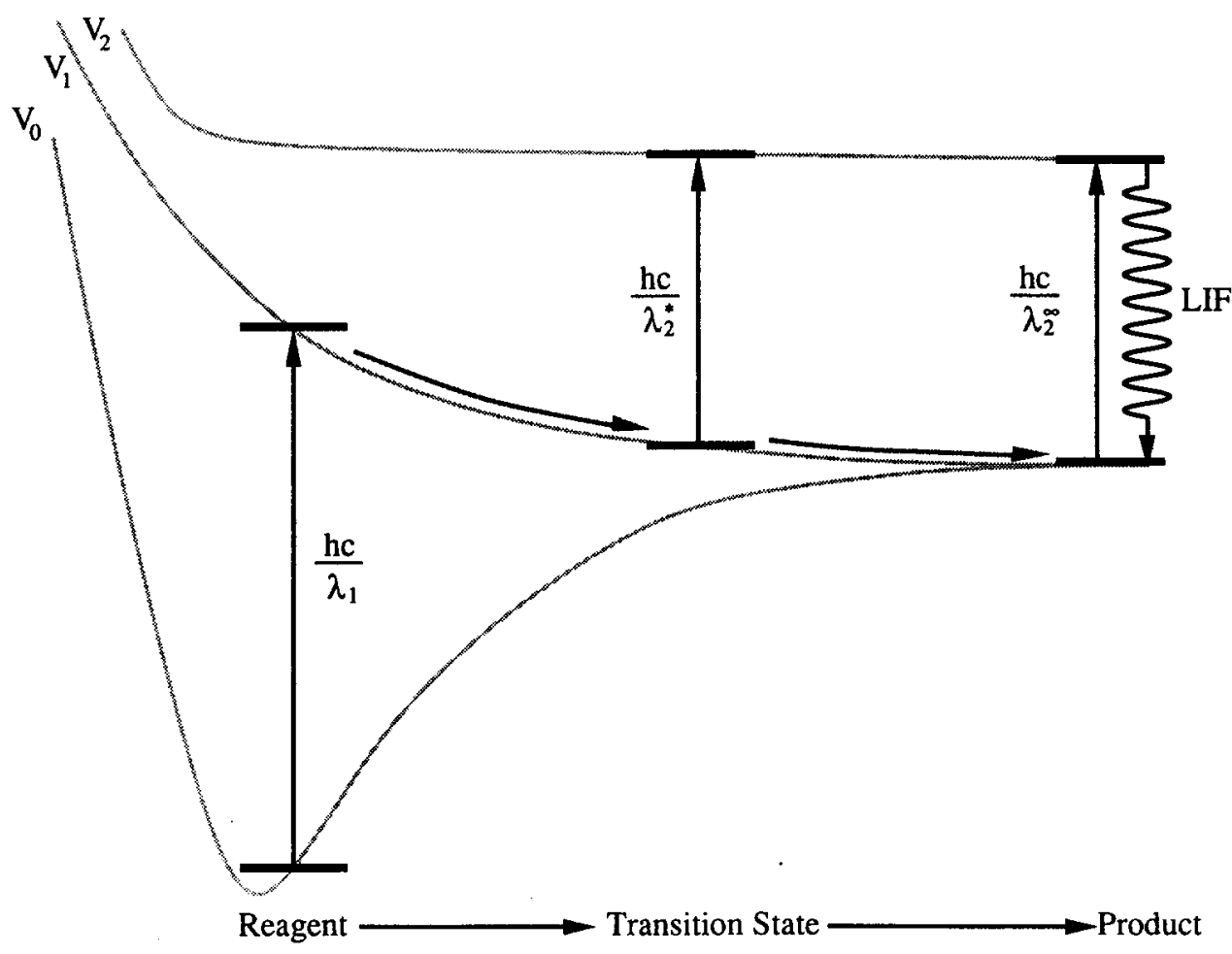

FIG. 1. Schematic representation of an FTS experiment. Three potential energy surfaces for an idealized reaction are shown. Absorption by the reagent molecule of the femtosecond pump pulse at wavelength $\lambda_{1}$ is associated with a vertical transition from the ground state $\left(V_{0}\right)$ to a repulsive surface $\left(V_{1}\right)$. Internuclear separation subsequently increases. After some delay time $\tau$, a probe photon at wavelength $\lambda_{2}^{*}$ (or $\lambda_{2}^{\infty}$ ) samples the absorption of the transition state (or final state) from $V_{1}$ to the next higher electronically excited surface $V_{2}$. The absorptivity of the probe is deduced experimentally through the collection of the laser-induced fluorescence (LIF) from $V_{2}$ to $V_{1}$.

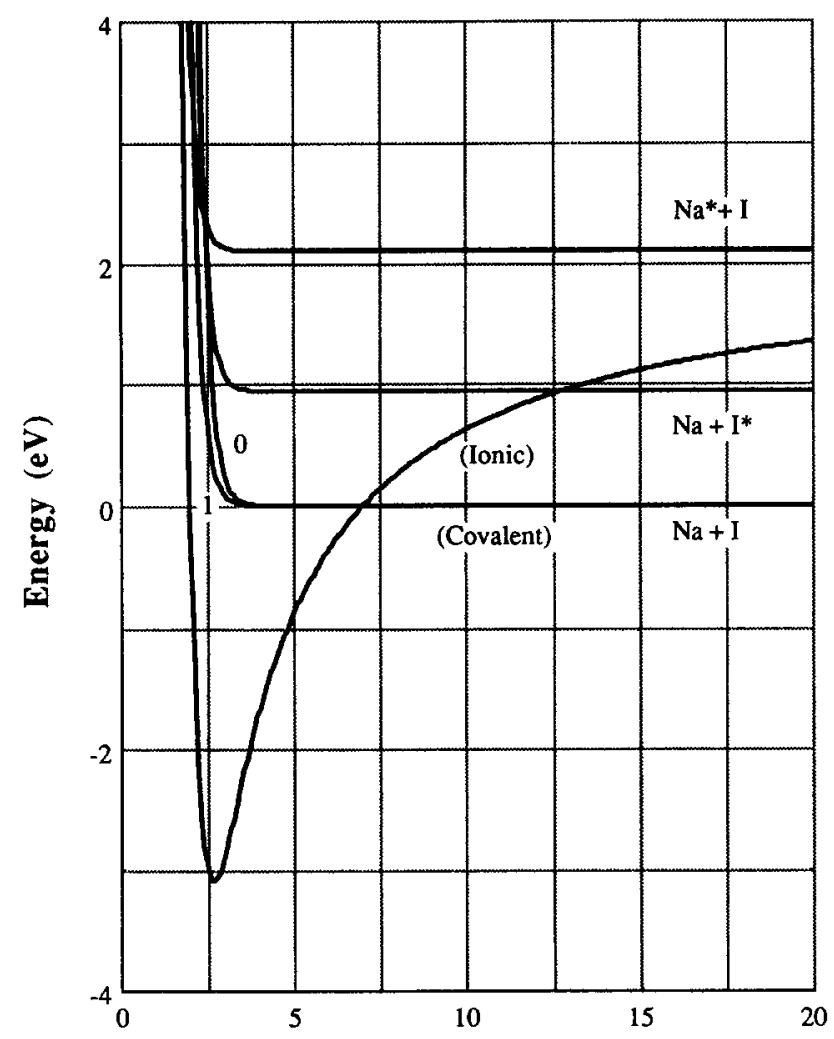

Separation ( $)$

FIG. 2. A schematic representation of the potential energy surfaces of NaI. The lowest energy ionic state $\left({ }^{\prime} \Sigma_{0}^{+}\right)$is $\mathrm{Na}^{+}+\mathbf{I}^{-} . \mathbf{N a}+\mathbf{I}$ is the lowest covalent state $\left[\mathrm{Na}\left({ }^{2} S_{1 / 2}\right)+\mathrm{I}\left({ }^{2} P_{3 / 2}\right)\right], \mathrm{Na}+\mathrm{I}^{*}$ is the next higher state $\left[\mathrm{Na}\left({ }^{2} S_{1 / 2}\right)+\mathrm{I}\left({ }^{2} P_{1 / 2}\right)\right]$, and $\mathrm{Na}^{*}+\mathrm{I}$ is the electronically excited sodium surface $\left[\mathrm{Na}\left({ }^{2} P\right)+\mathrm{I}\left({ }^{2} P_{1 / 2}\right)\right]$. Both covalent states are shown $\left(\Omega=0^{+}\right.$ and $\Omega=1$ ). to pure dissociative behavior, as occurs in ICN. However, when the interaction is relatively strong, a trapping well may be formed because of an avoided crossing. In this adiabatic limit the potential well delays the dissociating fragments and causes a resonance behavior.

One way to describe the degree of diabaticity is to use the Landau-Zener theory. ${ }^{13}$ This formalism employs a parameter $P_{\mathrm{LZ}}$ which represents the probability that a molecule will jump from one adiabatic surface to another as it makes a single pass through the interaction region. (Figure 3 illustrates this concept.) A $P_{\mathrm{LZ}}$ parameter close to unity, therefore, is indicative of diabatic behavior, while a small factor denotes an adiabatic tendency. This diabatic or adiabatic character plays a crucial role in the dissociative dynamics and in the FTS observations.

Both the energy level scheme and the degree of adiabaticity in the various alkali halides have been the subject of much experimental and theoretical investigation over the past 50 years. ${ }^{12,14-21}$ Absorption and emission, ${ }^{12,14,22-24}$ cross beam scattering, ${ }^{11,25,26}$ and photofragment spectrosco$\mathrm{py}^{19,20}$ have all been utilized, and evidence for bound excited states in $\mathrm{NaI}$ (and other alkali halides) have been presented. Theoretically, the coupling between the ionic and covalent surfaces has been calculated, ${ }^{15}$ and its connection to beam experiments was made. ${ }^{16}$ More recently, high resolution spectroscopy was invoked to analyze the vibrational structure of $\mathrm{NaI}^{*}$ and obtain information on the surface in the Franck-Condon region. Ragone et al. ${ }^{27}$ obtained the first jet-cooled spectra. Because of the complexity of the vibrational- rotational spectra and the cooling condition the analysis of the spectra was later reconsidered. ${ }^{28}$ In a subsequent high resolution study by Schaefer $e t$ al., ${ }^{21}$ a complete analysis, using the formalism of $\mathrm{Child}^{29}$ was made and the vibra- 


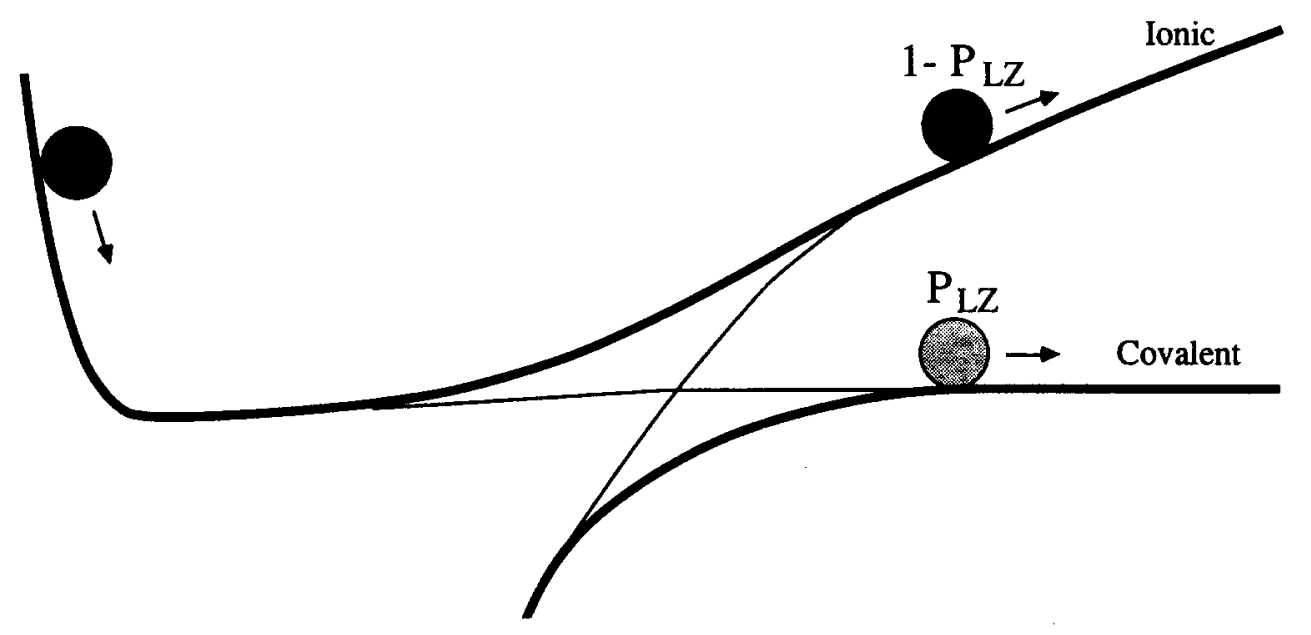

FIG. 3. Classical description of a diabatic level crossing. The wave packet (solid ball) encounters the interaction region from the left. The packet has probability $P_{\mathrm{LZ}}$ of transmission across this region to the other PES.

tional spacings were deduced. These experiments, and those by Delvigne and Los ${ }^{26}$ and by van Veen and co-workers ${ }^{19}$ have provided valuable information on the covalent potential parameters and the interaction between the covalent $0^{+}$ and ground ionic states.

Probing of the transition region of $\mathrm{NaI}$ was first attempted by the Polanyi group using the (time-integrated) "wing emission" method. ${ }^{17} \mathrm{~A}$ wing emission, down in intensity by approximately six orders of magnitude from that of $\mathrm{Na}^{*}$ emission, was reported and attributed to arise from the transition states. In a more recent publication, ${ }^{30}$ the group indicated that the presence of a van der Waals well in the upper state had resulted in a copious bound emission that masked the transition-state emission.

This paper provides a fuller account of our studies ${ }^{8,9}$ of the femtosecond real-time dynamics in the transition-state region of the NaI reaction using FTS. We detail observations relating to wave packet trapping dynamics and obtain the characteristic times for covalent-to-ionic motion, as well as the Landau-Zener probability for escaping diabatically. By varying the recoil velocity, we deduce characteristics of the PES and the escape probability as a function of total energy. As in our earlier communications, ${ }^{8,9}$ we use a classical mechanical model to describe the motion. We also compare with the recent quantum mechanical results of Engel et al., ${ }^{31}$ and make contact with many more recent theoretical studies of these FTS results by the groups of Light, ${ }^{32}$ Marcus, ${ }^{33}$ and Lin and Fain. ${ }^{34}$

The paper is organized in the following manner. Section II contains the experimental details of the FTS pertinent to the alkali halide experiments, while Sec. III gives the results and discussion. In Sec. IV, an analysis of the data is given in terms of classical and quantum mechanical models. Section V describes results obtained for some other alkali halides. In the final Sec. VI, the conclusions are given.

\section{EXPERIMENTAL}

The experimental methodology of FTS was presented in paper I of this series. ${ }^{1(a)}$ In this section, specific details pertinent to the FTS experiments of the alkali halide reactions are provided.

\section{A. Pump and probe generation}

The femtosecond pulses were generated from a colliding pulse mode-locked ring dye laser (CPM), and were amplified by a Nd:YAG-pumped pulsed dye amplifier (PDA). The output pulses from this system had $\sim 300 \mu \mathrm{J}$ energy, 20 $\mathrm{Hz}$ repetition rate, and were centered at approximately 620 $\mathrm{nm}$. From the autocorrelation traces of these PDA pulses, the temporal pulsewidth was measured to be as short as $60 \mathrm{fs}$ (assuming a hyperbolic secant-squared pulse shape).

The experiments required tunable radiation at the pump and the probe wavelengths. One generation technique is shown schematically in Fig. 4. In practice, a variety of frequency generation procedures were employed to convert a portion of the PDA output to the desired pump wavelength (Fig. 5). One of these techniques, which produced the shortest pump pulses, was second harmonic generation of the PDA light, accomplished by focusing the light into a thin $(500 \mu \mathrm{m})$, phase-matched KD*P crystal [Fig. 5(a)]. This technique was quite efficient ( $\sim 10 \%$ conversion), and yielded light at $\sim 310 \mathrm{~nm}$.

Since broad tunability was desired, we have also made use of ultrafast white-light continuum generation [Fig. 5 (b) $].{ }^{35}$ This was performed by tightly focusing the PDA light into a flowing $\mathrm{H}_{2} \mathrm{O}$ cell. An interference filter was used to select out the required spectral region from this continuum. The spectral bandpass of the interference filter was 100 $\AA$, and the central wavelength was tunable over a broad range by angular rotation of this filter. The light transmitted through this filter was further amplified in a flowing cell of laser dye transversely pumped by $100 \mathrm{~mJ}$ of $532 \mathrm{~nm}$ radiation from the same Nd:YAG laser that pumped the PDA. The selection of dye depended on the desired wavelength; in this study, the dyes used included Rhodamine 590, Kiton Red, Rhodamine 640, DCM, and LDS 698. Energies up to $\sim 75 \mu \mathrm{J}$ and wavelengths from 550 to $728 \mathrm{~nm}$ were produced in this way. The pump, 275 to $364 \mathrm{~nm}$, was generated by doubling this light, in the same manner as described above. Cross correlation with the PDA light revealed substantial temporal broadening of the pulse. Typical pulse durations were in the range of 150 to $200 \mathrm{fs}$.

A third technique, sum frequency generation (SFG), 


\section{Generation of Pump and Probe Pulses}

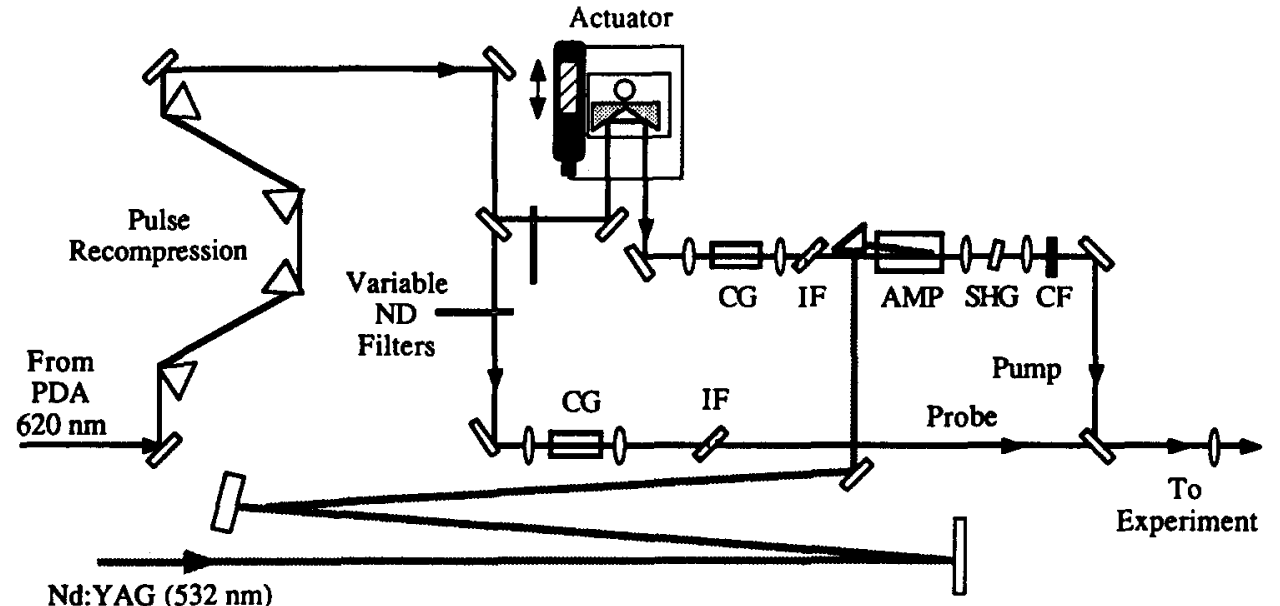

FIG. 4. Schematic representation of the pump/probe generation. Key: $\mathrm{CG}=$ continuum generation cell; IF = interference filter; SHG = second harmonic generating crystal; $\mathrm{CF}=$ color filter; $\mathrm{AMP}$ $=$ amplification stage. was used to further extend the tuning range [Fig. 5(c)]. Residual light from the Nd:YAG laser at $1.064 \mu \mathrm{m}$ was mixed together with the PDA output at $\sim 620 \mathrm{~nm}$ to produce light at $\sim 388 \mathrm{~nm}$. As has been previously demonstrated, (a) this procedure yields transform-limited pulses of approximately 100 fs duration.

The probe pulses measured the absorption of the system

(a)
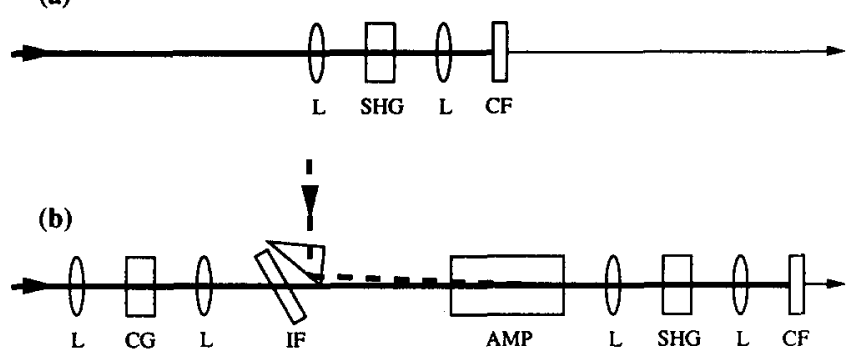

(c)

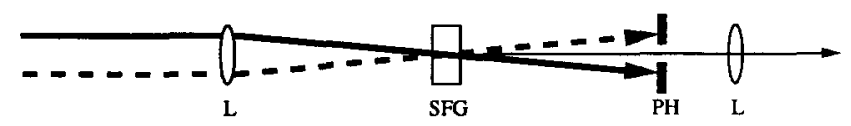

(d)

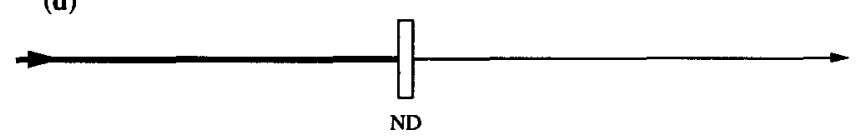

(e)

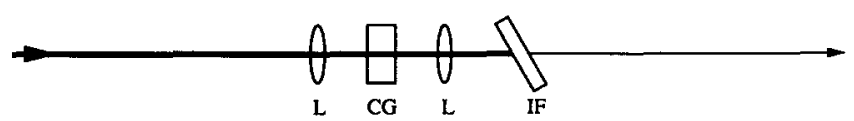

FIG. 5. Pump and probe generation schemes. Key: $L=$ lens; $S H G=$ second harmonic generating crystal; $\mathrm{CF}=$ color filter; $\mathrm{CG}=$ continuum generation cell; IF = interference filter; $A M P=$ amplification stage; SFG = sum frequency generating crystal; $\mathbf{P H}=$ pinhole. (a) Second harmonic generation; (b) continuum generation with amplification stage; (c) sum frequency generation; (d) direct use of pulsed dye amplifier output; (e) continuum generation. at (or off-resonant to) the $D$ lines of free $\mathrm{Na}$ at 589.0 and $589.6 \mathrm{~nm}$. Probe generation was performed using either of two approaches. The simplest "generation" method is illustrated in Fig. 5(d); in this case, the probe was obtained directly from the PDA output. A thin, Al-coated, neutral density filter was used for attenuation. While the shortest probe pulses could be obtained in this way, the wavelength was fixed to be that of the CPM laser, $\sim 620 \mathrm{~nm}$. Alternately, tunable pulses were produced by continuum generation [Fig. 5(e)]. Further amplification of these pulses was not required. The spectral bandpass of the interference filter was $10 \mathrm{~nm}$, although for certain experiments $1 \mathrm{~nm}$ filters were used (see below).

\section{B. Reaction compartment and data collection}

The pump and probe beams, with proper attenuation and either parallel or perpendicular polarization, were delayed in time relative to one another in a Michelson interferometer arrangement. The beams were colinearly recombined and focused into an $8 \mathrm{~cm}$ long, vacuum sealed quartz cell, which contained the sample (either $\mathrm{NaI}, \mathrm{NaBr}$ or $\mathrm{LiI}$ ). Prior to sealing, the sample (purity greater then $99.9 \%$ ) was heated at $400^{\circ} \mathrm{C}$ under vacuum ( $<10^{-6}$ Torr) for approximately $8 \mathrm{~h}$ to remove excess water. For the experiments, the cell was heated in an oven to a temperature of $\sim 650^{\circ} \mathrm{C}$. An attached sidearm of the cell was employed to control the vapor pressure of the sample. The sidearm was maintained at lower temperatures than the oven to avoid condensation on the cell windows. The vapor pressure of the sample was estimated from transmission measurements through the cell to be in the range of $100 \mathrm{~m}$ Torr. Temperatures approximately 50 to $100^{\circ} \mathrm{C}$ higher were employed for the $\mathrm{NaBr}$ sample because of its lower vapor pressure and absorption cross section. Deterioration of the $\mathrm{NaBr}$ cell (i.e., the material would coat out on the windows of the cell) was noted after about 10 $h$ of use. In more recent experiments we have used a heat pipe arrangement ${ }^{36}$ and the results from $\mathrm{NaI}$ were found to be essentially the same.

The laser-induced fluorescence (LIF) in a direction orthogonal to the pump/probe pulses was collected by an 
lens arrangement. Spurious light was also rejected by dispersing the LIF through a $0.34 \mathrm{~m}$ monochromator which was tuned to the free $\mathrm{Na} D$ lines $(589 \mathrm{~nm})$. The fluorescence, which showed the characteristic $\sim 16 \mathrm{~ns}$ decay, ${ }^{37}$ was detected with a Hamamatsu R1527 photomultiplier tube. The portion of the PMT voltage which occurred within an adjustable temporal gate was integrated by a digital boxcar integrator. A microcomputer controlled the optical delay line of the Michelson interferometer and collected the boxcar data. High signal-to-noise ratio was achieved by numerically averaging together up to 100 separate data sets. Typically, 20-30 scans were averaged.

Emphasis was given to fully characterize the pump and probe pulses. For each data set, the spectrum of the pump and the probe were taken. Also, the cross correlation of the pump with the probe was measured, using the technique of Type I difference frequency generation (DFG) in a $\mathbf{K D}^{*} \mathbf{P}$ crystal $\left(\omega=\omega_{\text {pump }}-\omega_{\text {probe }}\right)$. This approach was not always effective because the DFG frequency was nearly degenerate with that of the probe. Rejection of the probe light from the DFG signal was therefore difficult (impossible at certain wavelengths), but where possible was accomplished using either cutoff (color) filters or a $10 \mathrm{~nm}$ bandpass interference filter. As an additional check, the autocorrelation of the PDA output before the pump/probe generation was also measured. Unlike the ICN experiments, the exact zero-oftime (the delay at which the pump and the probe were temporally coincident) was not exactly determined in these experiments.

\section{Diagnostic experiments}

Numerous diagnostic experiments were performed. These include: (1) verification of signal linearity with respect to pump and probe intensity, (2) consideration of "background" effects (scattered light, two-photon absorption, etc.) and pressure dependence, (3) polarization studies, (4) wavelength tuning, and (5) bandwidth effects.

\section{Power dependence}

The effects of saturation (i.e., a significant deviation from linearity of the observed signal as a function of the pump or probe intensity) upon the shape of the FTS transient have been discussed elsewhere, using a kinetic model. ${ }^{1(a)}$ In simulations of FTS results on a repulsive potential, $^{1(\mathrm{~b})}$ for instance, the dominant effect of saturation by an on-resonant probe was found to be a shift in the transient to earlier delay times, but with very minor changes in the transient shape. In other simulations ${ }^{1(b)}$ (off-resonant), the transient shapes were affected by such saturation.

In the present study, for sufficiently high intensities of the pump or probe, the signal was found to saturate. To identify the regime for which the signal was linear with respect to the pump and probe intensities, two separate studies were made. First, at a fixed delay $(\sim 10$ ps after the initial rise), the dependence of the LIF signal on the pump or probe energy was recorded. This measurement was performed for both on-resonant $\left(\lambda_{\text {probe }}=589 \mathrm{~nm}\right.$ ) and off-resonant (typically, $\lambda_{\text {probe }}=620 \mathrm{~nm}$ ) probe wavelengths and at low to medium intensity levels. The results are plotted on a log-log scale in Fig. 6. All the data sets, except Fig. 6(c), yield a straight line with slopes near unity. A near-unity slope is consistent with a one-photon process; the $\mathrm{NaI}$ is excited by a single pump photon and the $\mathrm{Na}$ fluorescence is induced by a single probe photon. The exception to this power linearity occurred for the measurements involving the on-resonance signal versus the pump intensity [Fig. 6(c)]; substantial sublinearity was observed. At very low pump intensities, however, the signal did exhibit linear behavior. Further tests
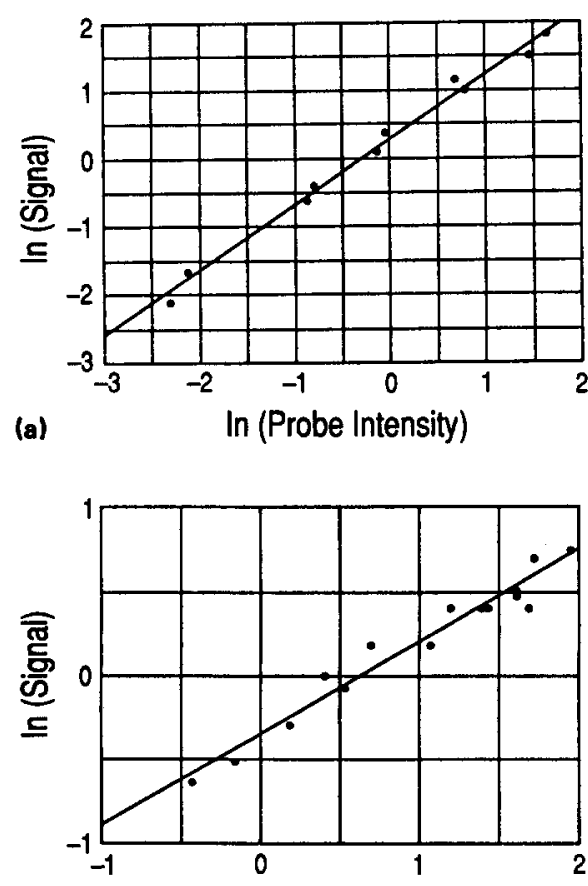

(c)

In (Pump Intensity)

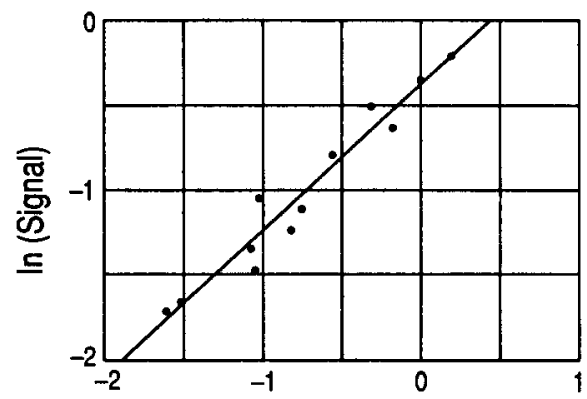

(b)

In (Probe Intensity)

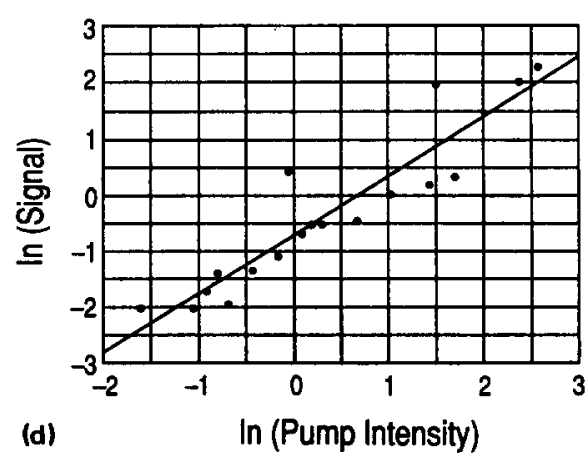

FIG. 6. Dependence of the signal on power with respect to: (a) (top left) probe intensity for on-resonant probe wavelength; (b) (top right) probe intensity for off-resonant probe wavelength; (c) (bottom left) pump intensity for on-resonant probe wavelength; (d) (bottom right) pump intensity for off-resonant probe wavelength. The solid circles are data, and the lines are best fits to a line. The slopes of the lines are: $0.96,0.86,0.55$, and 1.06 , respectively. 
of the signal power dependence involved the comparison of on- and off-resonance FTS transients taken over a wide range of pump and probe powers. The results of these measurements are discussed in Sec. III. It was concluded that for the typical experimental parameters, linearity of the signal with respect to both the pump and the probe was satisfactory, and the transient shape was independent of these intensities.

\section{Background signal level and pressure effects}

The pump and the probe each contributed a small background level to the observed data, which was independent of the delay between the pump and probe pulses. A part of this "DC" level was probably caused by weak multiphoton absorption of the NaI by the pump light; it was virtually eliminated by the suitable attenuation of the pump beam. A more significant contribution was associated with scattered probe light. Its level was minimized (to typically $\sim 5 \%$ of the transient peak level) by (1) careful orientation of the cell, (2) imaging of the interaction region on the slit of the monochromator, and (3) appropriate adjustment of the boxcar temporal gating. Finally, photons produced by thermal (blackbody) radiation from the $650^{\circ} \mathrm{C}$ cell were collected; this light was also suppressed by temporal gating, as the number of blackbody photons arriving within the nanosecond boxcar gate was negligible. It was verified that the transient shape was unaffected by these background contributions. ${ }^{38}$

In addition to the free $\mathrm{Na}$ fragments produced by the pump pulse, there is also a background $\mathrm{Na}$ population resulting from the thermal dissociation of $\mathrm{NaI}$. The amount of thermally produced $\mathrm{Na}$ at the ambient temperature of the cell was estimated to be well below $10^{10}$ atoms $/ \mathrm{cm}^{3} .^{39}$ For the experimental conditions (pump pulse energy, spot size, etc.), the concentration of the photoinduced species was estimated to be four orders of magnitude greater, since at the pressure used $(\sim 0.1-0.2$ Torr $)$, the $\mathrm{NaI}$ concentration was $\sim 7 \times 10^{15}$ molecules $/ \mathrm{cm}^{3}$. There was no evidence of any thermal contribution to the FTS transients, as the oven temperature was varied over a range of approximately $100^{\circ} \mathrm{C}$ without affecting the signal.

Since the average $\mathrm{NaI}$ hard sphere collision time at 100 mTorr and $650^{\circ} \mathrm{C}$ is $\sim 0.1 \mu \mathrm{s}$, the effect of collisions on the dynamics of the reaction (on the femtosecond time scale) or fluorescence (on the nanosecond time scale) can therefore be ignored. When the density of the sample was varied over an order of magnitude, no significant change in the transient behavior was observed.

\section{Polarization effects}

In previous FTS studies from this laboratory, femtosecond coherence and alignment have been investigated by changing the relative direction of polarization of the pump and probe beams. ${ }^{1(b), 40,41}$ As has been pointed out, ${ }^{41}$ a comparison of the transient shapes for the two polarizations yields information on the coherence time of the reaction. To check for polarization effects, we have verified that similar
FTS transients were obtained for both parallel and perpendicularly polarized pump and probe beams. For these experiments, the pump (double PDA light) and probe (direct PDA light) beams were generated as described in Fig. 5; continuum light was avoided because of the possibility of slight depolarization. In all cases the probe beam polarization was oriented along the direction of the LIF collection. The pump beam polarization was adjusted with the rotation of a zero-order, half-wave retardation plate. The results of this experiment are important in showing that the major features of the transients in both parallel and perpendicular polarizations are unchanged. However, quantification of the signal amplitude and their relationship to theory of reaction alignment $^{40,41}$ will be dealt with elsewhere.

\section{Wavelength tuning and bandwidth effects}

The various generation schemes described above afforded wide tunability of both the pump and the probe. Experiments of primary importance were the measurements of the FTS transients as a function of the wavelength of the pump and of the probe. The pump tuning experiments were made using the continuum generation/amplification scheme [Fig. $5(\mathrm{~b})]$. A single data point at $\lambda_{\text {pump }}=388 \mathrm{~nm}$ was also obtained with the frequency mixing approach of Fig. 5(c). For the on-resonant case, the probe was produced by continuum generation [Fig. 5(e)]. To obtain better temporal resolution, the off-resonant data was collected using PDA light as the probe [Fig. 5(d)].

The probe tuning experiments were performed with pump light at $307 \mathrm{~nm}$, derived from SHG of the PDA [Fig. 5 (a) ]. The probe light was produced by continuum generation. For the best off-resonance temporal resolution, continuum generation was avoided; the pump and probe were obtained from the second harmonic and fundamental of the PDA, respectively. With all these different schemes, the results were reproducible (the same resonance frequency was always obtained for any given pump wavelength).

A study was also made of the effects of changing the spectral and temporal bandwidths of the pump or probe on the observed transient. For the case of the probe, tuning measurements were repeated using a $1 \mathrm{~nm}$ (instead of 10 $\mathrm{nm}$ ) bandwidth interference filter after the continuum. Significant broadening of the pulse was expected, as the transform limited width corresponding to a $1 \mathrm{~nm}$ pulse centered at $589 \mathrm{~nm}$ is $\sim 400 \mathrm{fs}$, whereas it is $\sim 40 \mathrm{fs}$ for a $10 \mathrm{~nm}$ width. These experiments were motivated by a desire to improve the spectral resolution of the experiment in order to determine the wavelength at which the off-resonance oscillations manifest themselves. Additionally, a comparison between short and long pulses was made to determine the effect of the pump characteristics (i.e., $\Delta v \Delta t$ ) on the dissociation dynamics. For these measurements, the pump and probe were obtained from the second harmonic and fundamental of the PDA. The pulse durations were varied by purposely changing the amount of dispersion in the CPM via the four compensating prisms. The results will be discussed in the following section. 


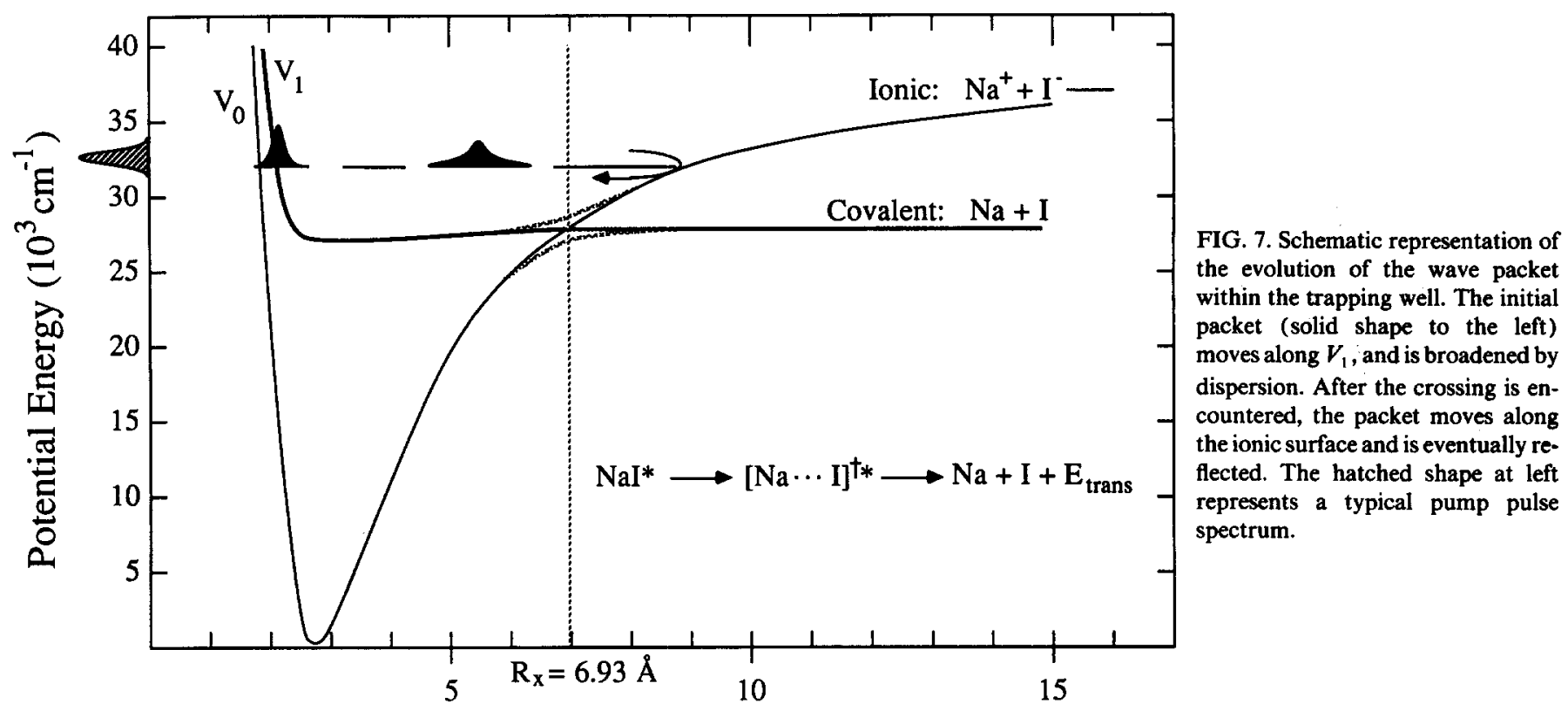

Internuclear Separation $(\AA)$

\section{RESULTS AND DISCUSSION}

\section{A. Preliminaries}

A simple description of the wave packet dynamics for the alkali halide reaction has been introduced previously. ${ }^{8,9}$ Figure 7 is illustrative of the evolution of the wave packet within the upper state adiabatic well for the case of $\mathrm{NaI}$. Absorption of the pump photon by the salt is associated with a vertical transition from the ionic to the covalent surface $\left(V_{0} \rightarrow V_{1}\right)$. The wave packet at $t=0$ has a nonzero spatial width, which is determined by the temporal and spectral widths of the optical pump pulse and the shape of the PES. As the molecule falls apart, it encounters the level crossing of these two surfaces. In the diabatic limit, the wave packet will transit this crossing with unity probability and will continue on to the product states. The observed FTS transients will in this case, as noted above, resemble those obtained for ICN.

In the adiabatic extreme, however, the wave packet may be trapped within the potential well formed by the interacting PESs. The temporal behavior in this case would be characterized by a strong resonance as the wave packet would move periodically within the potential well. Since the well is anharmonic, the packet would be dispersively broadened (see Fig. 7); eventually, the packet can be expected to uniformly fill the well.

The actual behavior of an alkali halide can be expected to fall between these diabatic and adiabatic extremes. We would therefore expect that the FTS results would give the shape of and the coupling between the potential surfaces.

There are various experimental parameters which can influence the dynamics of the MX dissociation reaction and help us deduce the PES characteristics. In the remainder of this section, the results of changing the pump and probe wavelength, bandwidth (pulse duration), and power are presented. These results will be considered within the framework of this basic model. Particular focus is placed on the reaction of $\mathrm{NaI}$. Later in this paper (Sec. IV), we will present a more formal theoretical treatment.

\section{B. Tuning the probe}

By varying the probe wavelength, one can effectively view the dynamics of the dissociative process at different positions along the reaction coordinate $(R) .^{1(c), 42}$ For example, when the probe is tuned to $589 \mathrm{~nm}$, it is resonant with the sodium $D$-line transitions (see Fig. 2). These transitions occur at very large (essentially infinite) values of $R$. Consequently, the fluorescence obtained under this condition is sensitive to the appearance of free sodium atoms $\left(\lambda_{2}^{\infty}\right.$ probing). If the probe is tuned either to the red or the blue of 589 $\mathrm{nm}\left(\lambda_{2}^{*}\right.$ probing), the resultant fluorescence originates from a species in a transition state.

A comparison of the results for on- and off-resonance tuning with a $10 \mathrm{~nm}$ bandwidth probe is shown in Fig. 8. The on-resonance signal [Fig. 8(c)] shows an initial sharp rise with a series of stair-stepped plateaus. Off resonance [Figs. $8(\mathrm{a}), 8(\mathrm{~b})$, and $8(\mathrm{~d})]$, the signal displays a damped oscillation, whose frequency matches that observed in the on-resonance case. For the data displayed in the figure, the pump (generated by doubling the amplified CPM output) was centered at $\lambda=307 \mathrm{~nm}$, had a bandwidth of $3 \mathrm{~nm}$, and had a pulse duration of $\sim 50 \mathrm{fs}$ (as determined by autocorrelation). The tunable probe was obtained from the passage of a generated continuum through an appropriate $10 \mathrm{~nm}$ bandwidth filter and possessed a pulse duration of approximately $250 \mathrm{fs}$.

\section{Off-resonance FTS: $\lambda$; probing}

In Fig. 8(b), the probe is off resonance, but not far enough to entirely eliminate the on-resonance contribution. Consequently, the signal does not decay to a level comparable to the signals at 570 and $615 \mathrm{~nm}$ [see Ref. 1(c)]. As 


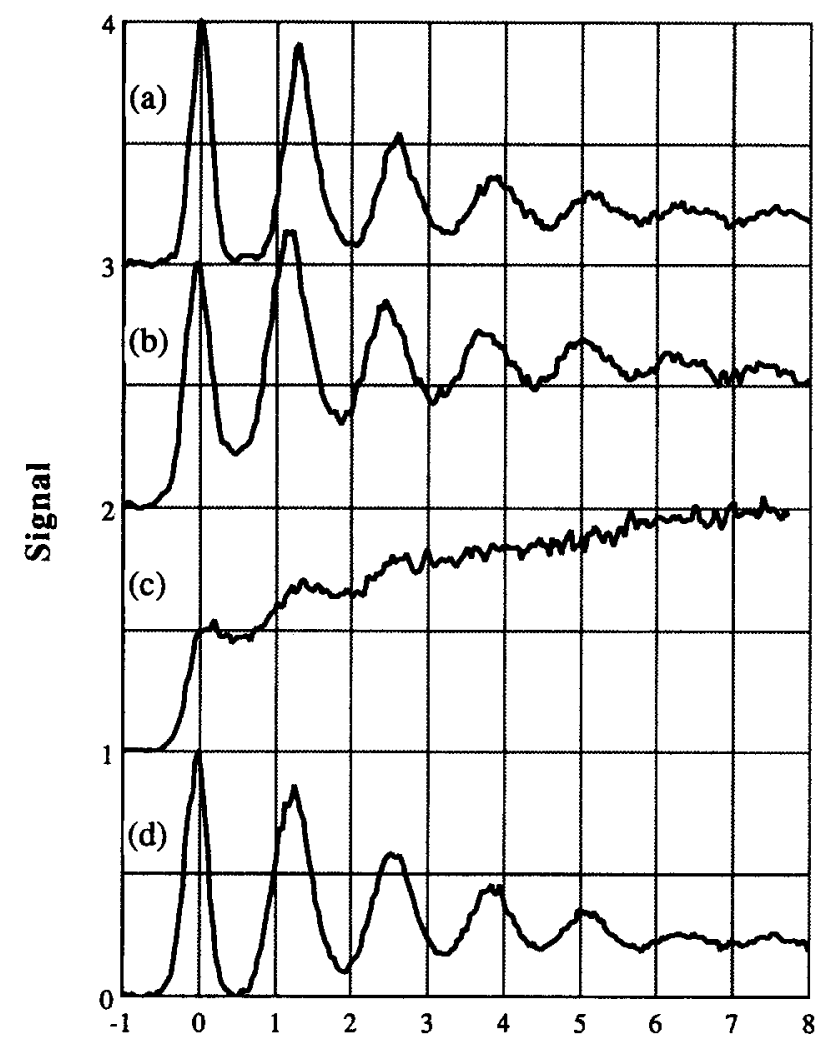

Time Delay (ps)

FIG. 8. FTS results for NaI as a function of probe wavelength. Key: (a) 575 $\mathrm{nm}$; (b) $580 \mathrm{~nm}$; (c) $590 \mathrm{~nm}$; and (d) $615 \mathrm{~nm}$. In this and all subsequent data figures, both the "zero of time" and the overall signal amplitude have been arbitrarily chosen.

described above, the oscillation is a manifestation of the propagation of the wave packet (initially generated by the pump) back and forth across the well. The period of this oscillation is on the order of $1 \mathrm{ps,} \mathrm{corresponding} \mathrm{to} \mathrm{a} \mathrm{frequen-}$ cy of approximately $1 \mathrm{THz}$.

Far off resonance, either to the red or the blue, the shape of the FTS transient was found to be essentially independent of the probe wavelength. Figure 9 compares the results obtained over a large wavelength range (see also Fig. 10). The effective tuning range for the probe for which signal could be obtained was 550 to $787 \mathrm{~nm}$. The red extreme for the experiment was determined by pulse generation considerations, while the blue edge was limited by the intrinsic absorption of the probe by the NaI. Thus, it is interesting to note that the off-resonance tuning extended much further to the red than to the blue. This behavior in tuning range is a function of the differences between the two excited potentials involved in the transition and can be used to deduce the potential difference change with time [see Ref 1 (c) ]. In an attempt to improve the spectral resolution for the probe detection, we used a narrower bandwidth filter. The data illustrated in Fig. 11 was taken with a tunable $1 \mathrm{~nm}$ bandwidth probe. The signalto-noise was not as good as in the broad band case and we are planning further experiments to obtain this change in the potential. $^{43}$

If absorption of the probe takes place from a unique

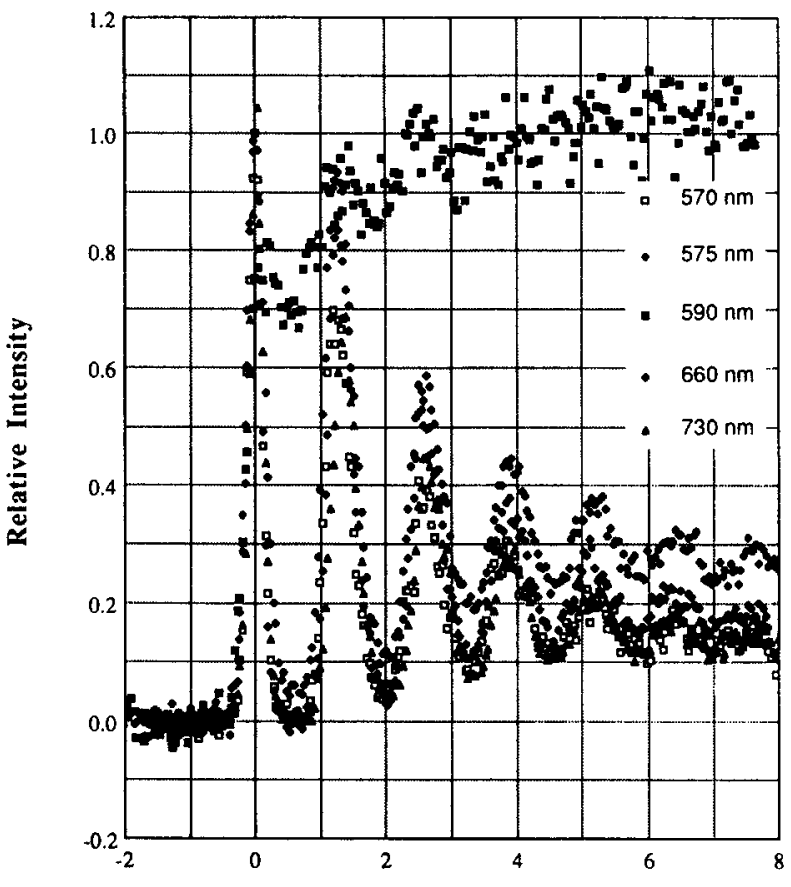

Time Delay (ps)

FIG. 9. FTS results for NaI for a broad range of probe wavelengths.

position along the reaction coordinate, two peaks, which will not in general be equally spaced, should occur per vibrational period; the wave packet enters the optically coupled region twice per cycle. An attempt was made to look for additional peaks or an inequality between the peak and trough widths by improving the temporal resolution of the experiment. This was accomplished by using the fundamental of the PDA for the probe, thus avoiding continuum generation altogether. Figure 10(a) shows that despite the improved temporal resolution ( $100 \mathrm{fs})$, no additional structure is revealed. In Fig. 10(b), the results of part (a) are expanded to show the relative widths of the first peak, second peak and second trough. The width of the first peak is $300 \mathrm{fs}$, while that of the second peak is $520 \mathrm{fs}$. Furthermore, the width of the second trough is roughly $500 \mathrm{fs}$. It is possible that with better temporal resolution we may be able to resolve more structure. In Sec. IV, a classical model for the FTS experiments is presented which addresses these issues and reveals the reasons for the observed transients.

Two important parameters which fully describe the dynamics can now be obtained: the oscillation time $\tau_{0}$ and the damping time $\tau_{d}$. Both are evident in the FTS transients. For example, at $\lambda_{\text {pump }}=311 \mathrm{~nm}$ (corresponding to a total energy above dissociation of $7200 \mathrm{~cm}^{-1}$ ), $\tau_{0} \approx 1.2 \mathrm{ps}$ and $\tau_{d}$ $\approx 5.4 \mathrm{ps}$. These parameters change with total energy. We shall use these results in the coming section to deduce characteristics of the PES and the Landau-Zener coupling.

\section{On-resonance FTS: $\lambda_{2}^{\infty}$ probing}

For the on-resonant experiment, the probe is tuned to the free $\mathrm{Na} D$-line transitions. (Note that the spectral band- 


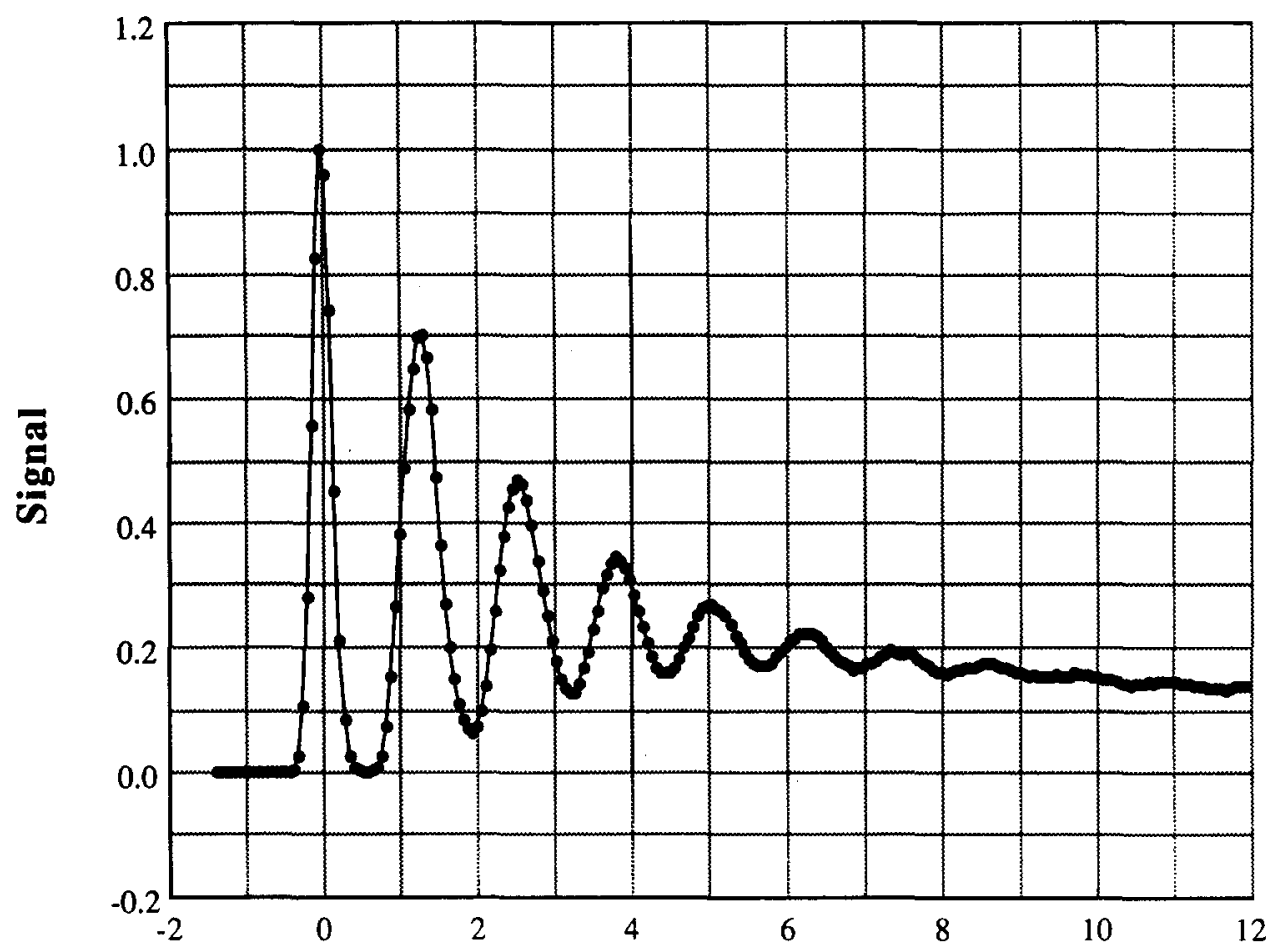

(a)

Time Delay (ps)

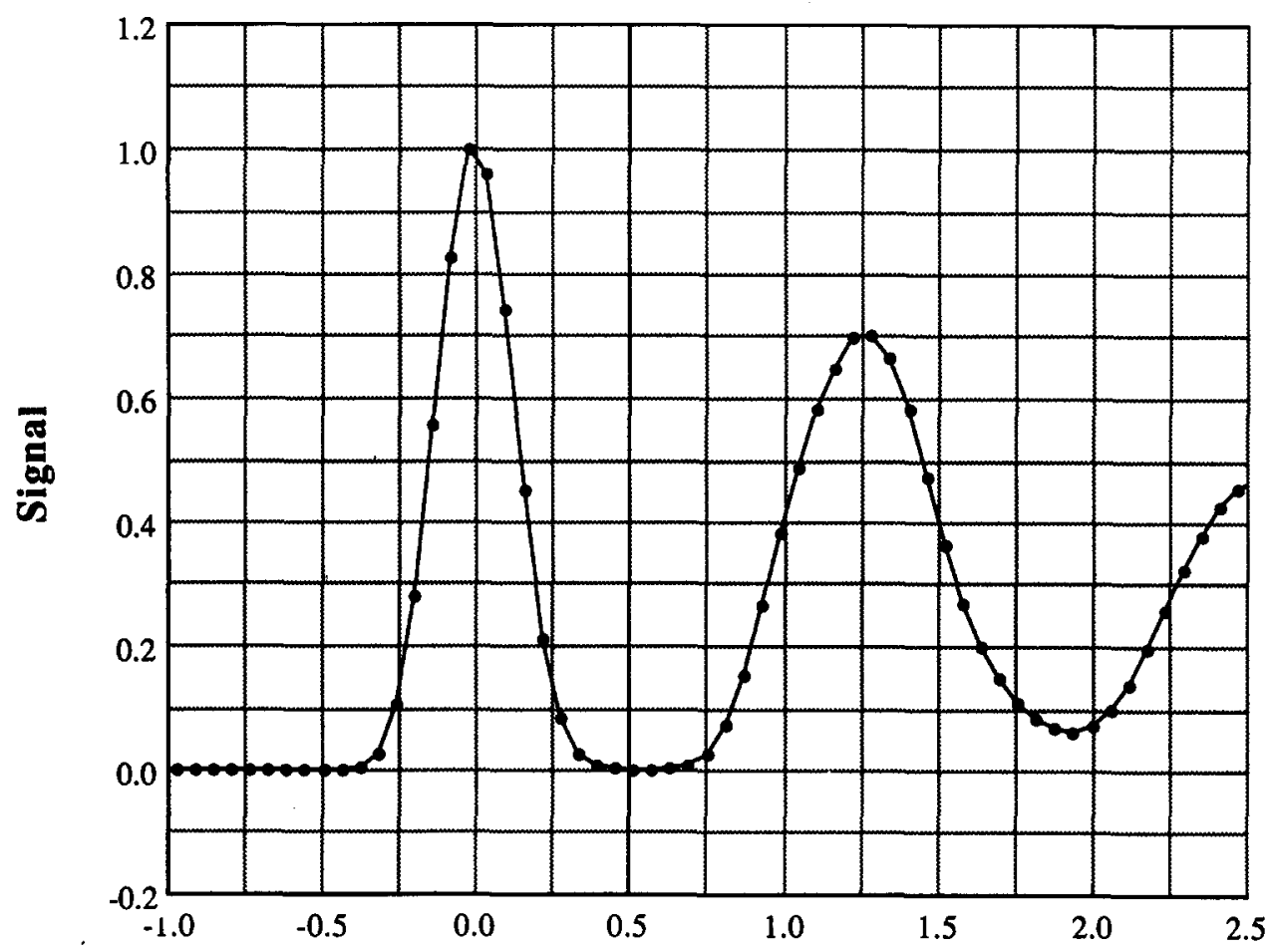

(b)

Time Delay (ps)
FIG. 10. FTS results for NaI with 100 fs resolution. An expanded scale is shown in (b), where the increase in width of the second peak relative to the first is apparent. The estimated full width of the first and second absorption peaks are 300 and $520 \mathrm{fs}$, respectively. width of the pulse is much larger than the $6 \AA$ separation between the two lines of the Na doublet. Furthermore, these lines are not resolved in the LIF detection.) The on-resonance signal shows an initial sharp rise followed by a series of rising plateaus which show the same temporal separation as the peaks in the off-resonance data, as expected in FTS. ${ }^{7}$ The probe detects the leakage of the wave packet each time it passes through the interaction region and effectively integrates the time dependence of the $\mathrm{Na}$ atom population. (Note that because of the wide spectral bandwidth of the probe, a slight dipping of the plateaus is observed due to the contribution of the off-resonance signal. That is, the experi- 


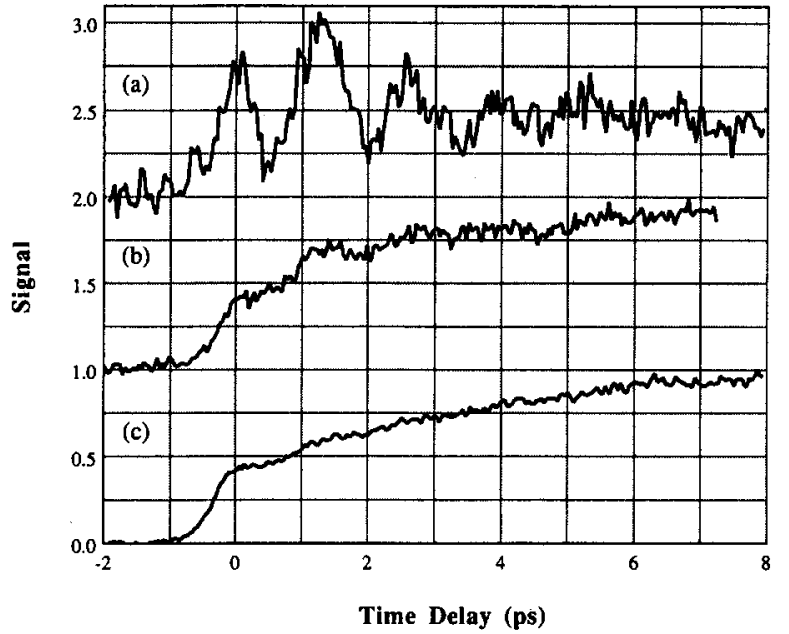

FIG. 11. FTS results for NaI with a $1 \mathrm{~nm}$ bandwidth probe pulse. Key: (a) $\lambda_{\text {probe }}=584 \mathrm{~nm}$; (b) $\lambda_{\text {probe }}=588 \mathrm{~nm}$; and (c) $\lambda_{\text {probe }}=590 \mathrm{~nm}$. Both the on-resonance and off-resonance transients have the same form as obtained with spectrally broader probe pulses.

mental results are a combination of the idealized on- and offresonant behavior.) In essence, the on- and off-resonance data should be complementary measurements of the dynamics of the system.

The rather large initial rising edge of the on-resonance scan does not correlate with the decrease in height between the first and second peak of the off-resonance data. This result suggests that there is a contribution to the on-resonance signal that is not detected off resonance. There exists a second channel by which the $\mathrm{Na}$ atoms may dissociate. This second pathway corresponds to the $\Omega=1$ potential energy surface $^{12}$ which is optically and energetically accessible from the ground state. Since this second state possesses a different symmetry than the ground state, there is no interaction and no avoided crossing region. Consequently, this surface is purely dissociative and allows for the rapid escape of $\mathrm{Na}$ atoms. As a result, the observed plateaus (dissociation via the $0^{+}$state) occur on top of a background signal (dissociation via the 1 state). On the other hand, the contribution of this channel to the off-resonance signal is less obvious in this pump wavelength regime (possibly due to lifetime effects); its effect would be the increase in magnitude of the first peak.

The competition between the two dissociative pathways as a function of energy has been well documented in the literature. By performing photofragmentation experiments, van Veen and co-workers ${ }^{19}$ have recently measured the relative contribution of the $0^{+}$and 1 states to the photofragmentation process as a function of excitation energy; the energy range explored was from 300 to $337 \mathrm{~nm}$. At a wavelength of $307 \mathrm{~nm}$, the relative contribution of the $0^{+}$ and 1 states was found to be approximately $1: 3$. The onresonance data [Fig. 8(c)] shows that magnitude of the fast component of the signal is roughly equal to the final magnitude of the slow component. It appears that both states contribute equally at the pump wavelength of $307 \mathrm{~nm}$. However, the nature of the packet prepared in the two experiments is different; therefore, there is a need to quantify the contribution of the $\Omega=1$ (repulsive) state under different experimental conditions. ${ }^{44}$ Later, we will discuss some interesting power dependences for these on-resonance transients.

\section{Tuning the pump}

By tuning the pump wavelength, one is changing the total available energy for the reaction, thereby preparing different wave packets on the covalent potential. If the trapping well is harmonic, the period of oscillation will be the same at all energies. However, if the well is anharmonic, the dissociation dynamics should exhibit a strong pump wavelength dependence. Thus, the FTS transients resulting from excitation to the bottom of the well should appear different from those scans taken with several thousand wave numbers of excess pump energy. In Figs. 12 and 13, typical off-resonance scans taken with pump wavelengths ranging from 300 to $364 \mathrm{~nm}$ are compared. (Note that the bottom of the well is estimated to be around $400 \mathrm{~nm}$.)

There are two distinct effects which correlate with tuning the pump wavelength to the red (that is, with decreasing the available energy for the reaction): (1) The oscillation period decreases, and (2) the damping (excluding the first peak) decreases. Interestingly, if the apparent increase in the

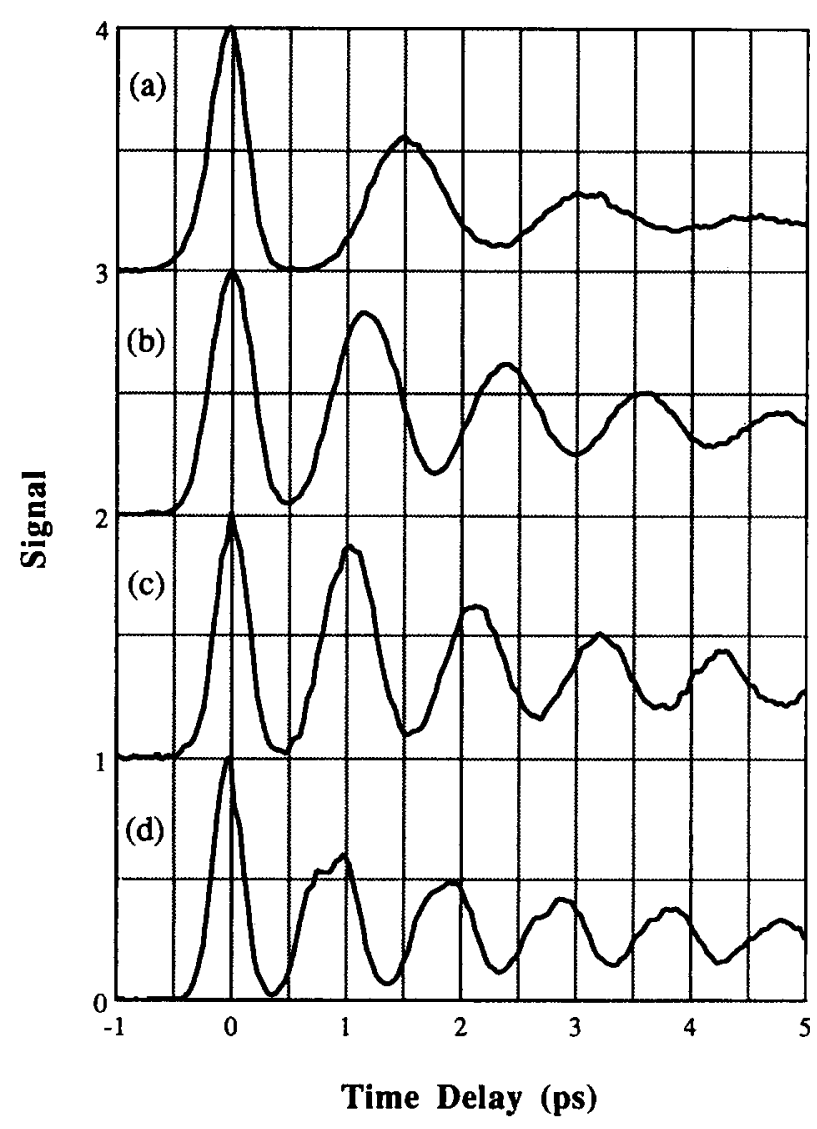

FIG. 12. FTS results for $\mathrm{NaI}$ for the tuning of the pump wavelength. Key: (a) $\lambda_{\text {pump }}=300 \mathrm{~nm}$; (b) $\lambda_{\text {pump }}=311 \mathrm{~nm}$; (c) $\lambda_{\text {pump }}=321 \mathrm{~nm}$; and (d) $\lambda_{\text {pump }}=339 \mathrm{~nm}$. The period becomes shorter as the wavelength of the pump is tuned to the red. Note that the damping changes as well, with the oscillations persisting longer for red wavelengths. 


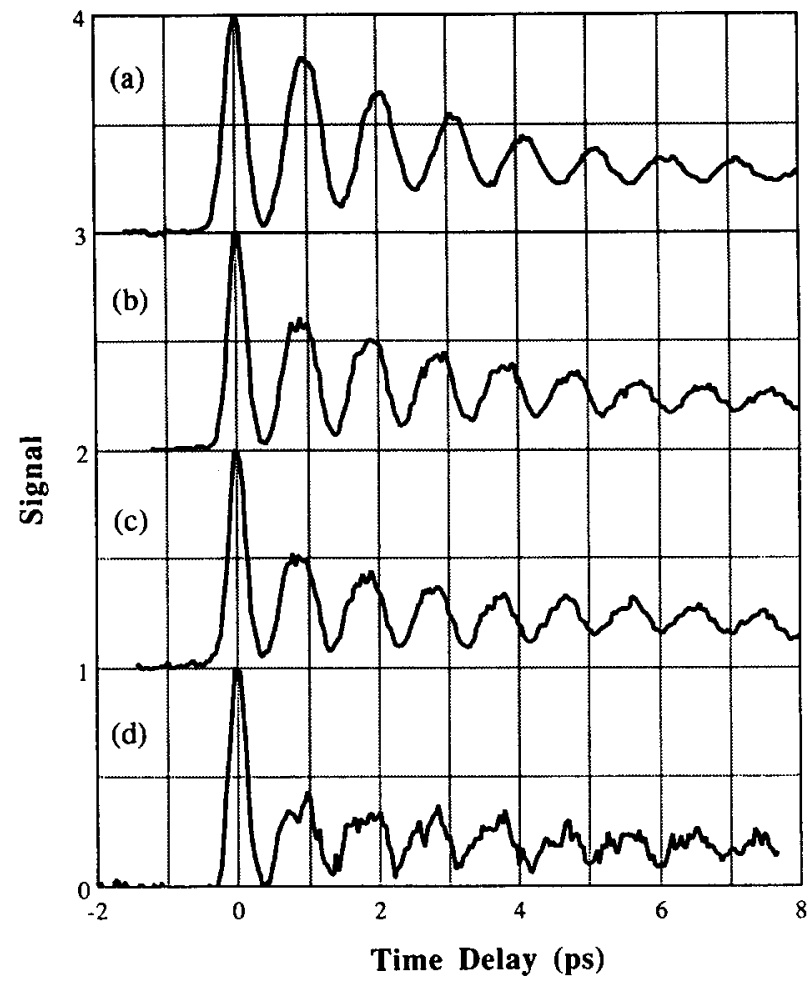

FIG. 13. FTS results for NaI for extremely red pump wavelengths. Key: (a) $\lambda_{\text {pump }}=332 \mathrm{~nm}$; (b) $\lambda_{\text {pump }}=344 \mathrm{~nm}$; (c) $\lambda_{\text {pump }}=354 \mathrm{~nm}$; and (d) $\lambda_{\text {pump }}$ $=364 \mathrm{~nm}$. In this range, the period is nearly independent of the pump frequency.

intensity of the first peak ${ }^{44}$ is due to the increased contribution of the $\Omega=1$ state to the dissociation of the molecule, then the result is in agreement with the work of van Veen et $a l .{ }^{19 \text { (a) }}$ (see Sec. III B 2).

\section{The nature of the trapping well}

Figure 14 displays a plot of the oscillation frequency (and period) versus the pump wavelength. The fact that the oscillation time increases as the energy increases indicates that the trapping well is anharmonic. As an initial model for the anharmonicity, one could start with the Morse potential which yields energy levels given by

$$
E_{n}=h v_{0}(n+1 / 2)-\chi h v_{0}(n+1 / 2)^{2},
$$

where $v_{0}$ is the standard harmonic oscillator frequency and $\chi$ is the anharmonic constant. It is easily shown (see Appen$\operatorname{dix} A$ ) that the frequency of the wave packet oscillation $\nu_{\text {packet }}$ can be expressed as follows:

$$
v_{\text {packet }}^{2}=v_{0}^{2}-4 \chi v_{0}\left(v_{\text {pump }}-v_{\text {diss }}\right) \text {. }
$$

In Eq. (5), $v_{\text {pump }}$ is the pump frequency and $v_{\text {diss }}$ is the dissociation frequency of the molecule. A plot of $v_{\text {packet }}^{2}$ vs ( $\nu_{\text {pump }}$ $\left.-v_{\text {diss }}\right)$ is shown in Fig. 15. The plot is linear over about two-thirds of the range wavelength range examined (i.e., at large excess energy). Nonlinearity occurs towards the red end of the tuning, i.e., towards the bottom of the well. Considering the Morse-type behavior in the linear portion of the data yields a value of $1.2 \times 10^{-3}$ for $\chi$. In this region, $n$ is large $(n \sim 180)$. This is in good agreement with the approximate value of $1.0 \times 10^{-3}$ for $\chi$ calculated from the data of Ref. 21, for this range of $n$. From our data, we see that the period changes with $n$, and $n$, of course, determines the frequency (e.g., for $n=180$, the vibrational frequency is $\approx 29$ $\mathrm{cm}^{-1}$ ). The oscillation frequency appears to approach a maximum value (and may even be decreasing) at the red end or towards the bottom of the well. This perhaps implies that the well changes from an anharmonic form (resulting from the $R^{-1}$ dependence of the Coulombic surface) to one which is more like a "particle in a box." Therefore, it would

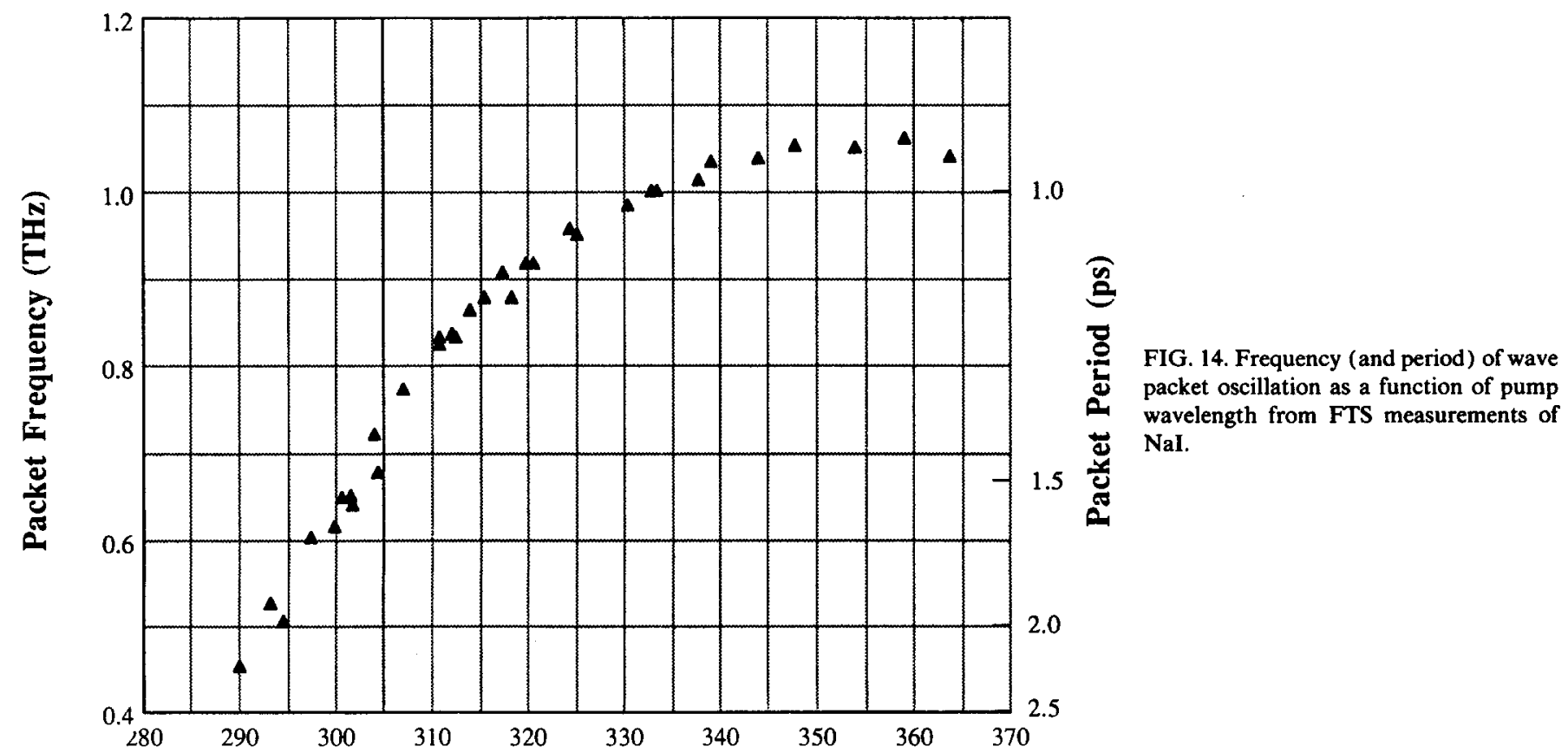

Pump Wavelength (nm) 


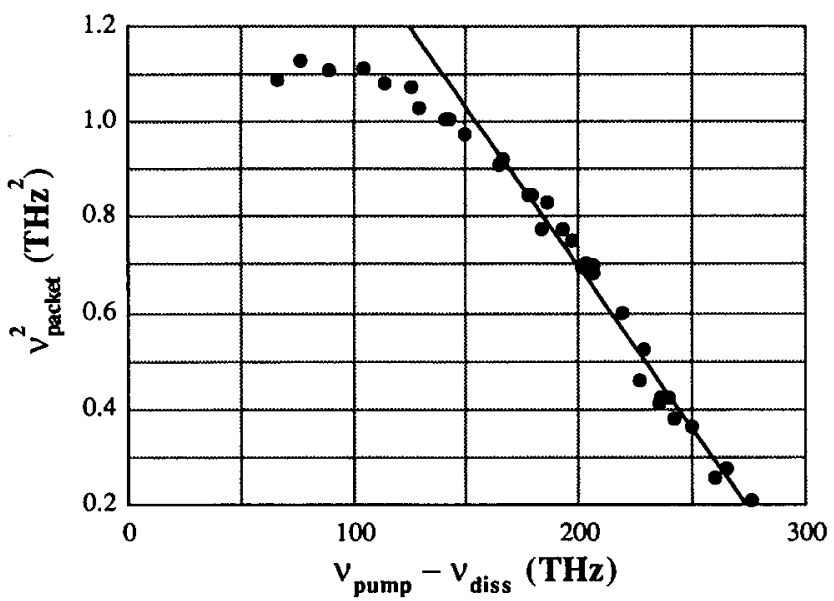

FIG. 15. The square of the observed frequency of the wave packet oscillation as a function of the laser pump frequency minus the frequency corresponding to the dissociation energy. (No correction to the thermal energy is made.) The line is a best fit to a portion of the data. At low excess energies, the relationship is no longer linear (see text).

be interesting to extend the pump wavelength further toward the bottom of the well. In this analysis we have not considered the initial vibrational population of the ground state, which will be dealt with later.

\section{The escape probability and covalent/ionic coupling}

The second effect of tuning the pump wavelength is the change in the damping time of the oscillations. One will note that the oscillations damp more quickly as one tunes to the blue. The Landau-Zener formula,

$$
P_{\mathrm{LZ}}=\exp \left\{-\frac{4 \pi^{2}}{h v_{r}(E, J)} \frac{V_{01}^{2}}{\frac{d}{d R}\left|V_{00}-V_{11}\right|}\right\}
$$

predicts that the probability of a molecule jumping from one potential surface to another is a function of the relative velocity of the particles at the crossing region. In Eq. (6), $V_{01}$ is the coupling matrix element between the two surfaces, the derivative gives the difference in slopes of the surfaces at the critical point, and $v_{r}(E, J)$ is the recoil velocity which is a function of the total energy $E$ and rotational quantum number $J$. Thus, for high pump energies, the recoil velocity term will be larger, $P_{\mathrm{Lz}}$ will be closer to unity and the damping of the observed oscillations should be greater. The general trend of the data follows the prediction that the higher the available energy, the larger the escape probability.

For illustration, we now calculate $P_{\mathrm{Lz}}$ at one available energy. From the data, at $\lambda_{\text {pump }}=311 \mathrm{~nm}$, the "recoil velocity" is $3 \times 10^{5} \mathrm{~cm} \mathrm{~s}^{-1}$. The decay envelope of our transient gives an escape probability of $11 \%$, i.e., $P_{\mathrm{Lz}}=0.11$. Since the covalent potential is essentially fiat at the crossing point, the difference in slopes is due to the Coulombic potential. From the value ${ }^{16}$ of $d V_{00} / d R \sim 5 \times 10^{-5} \mathrm{erg} / \mathrm{cm}$, we deduce that the coupling matrix element is $370 \mathrm{~cm}^{-1}$. This is in agreement with the calculation of Ref. 15 and with the experimental values obtained from chemi-ionization and spectroscopic analyses. ${ }^{21}$ Refinement of the $V_{01}$ value will be achieved when the contribution of the $\Omega=1$ state to the dynamics and the rate of spreading of the wave packet is

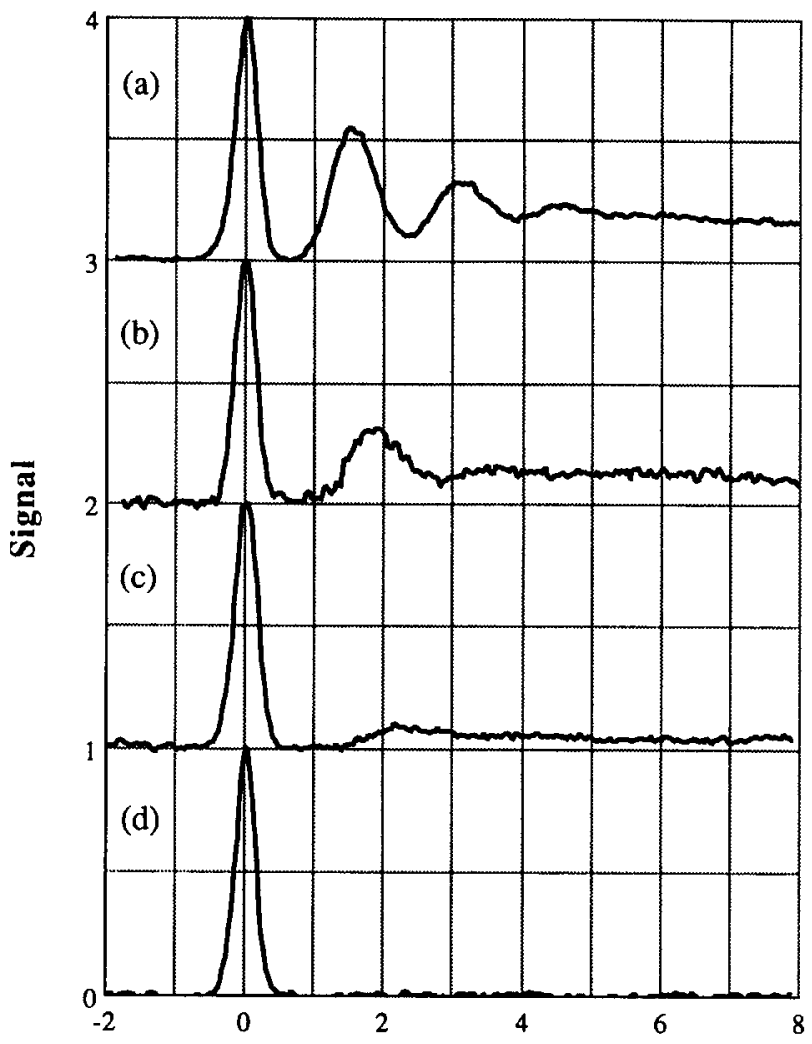

Time Delay (ps)

FIG. 16. FTS results for NaI for short pump wavelengths. Key: (a) $\lambda_{\text {pump }}$ $=300 \mathrm{~nm}$; (b) $\lambda_{\text {pump }}=295 \mathrm{~nm}$; (c) $\lambda_{\text {pump }}=290 \mathrm{~nm}$; and (d) $\lambda_{\text {pump }}=284$ nm.

quantified (see Sec. III B 2).

In Fig. 16 it can be seen that the oscillations are more dramatically damped when the pump is to the blue of 300 nm, where the experimental values of $P_{\mathrm{Lz}}$ from the data appear to approach a maximum value. A single peak is observed for pump wavelengths less than $285 \mathrm{~nm}$ (in the time range studied). This may be the result of the new $\mathrm{Na}+\mathrm{I}^{*}$ channel. As suggested by the work of Davidovits and Broudhead, ${ }^{45}$ absorption leading to the dissociation of $\mathrm{NaI}$ into ground state sodium and spin-orbit excited iodine $\left(I^{*}\right)$, starts to occur in the wavelength region around $285 \mathrm{~nm}$. At longer wavelengths both atomic fragments are created in their ground states. The potential energy surface resulting in the formation of ground state $\mathrm{Na}$ and of $\mathrm{I}^{*}$ crosses the ground state surface at a distance of $\sim 13 \AA$.

\section{Power dependence}

In Sec. II, the power dependence of the FTS transients were analyzed at a fixed time delay. It was found that the signal intensity was essentially linear with respect to the pump and probe pulse intensities (over a reasonable range) except for the case of the on-resonance pump dependence. In order to further study the effects of the pulse powers on the signal, entire FTS transients for various pump and probe intensities are compared. For the off-resonant case (Fig. 17), the transients were remarkably consistent in shape over factors of $\mathbf{3 0}$ for the pump and $\mathbf{1 0 0}$ for the probe. Similarly, 


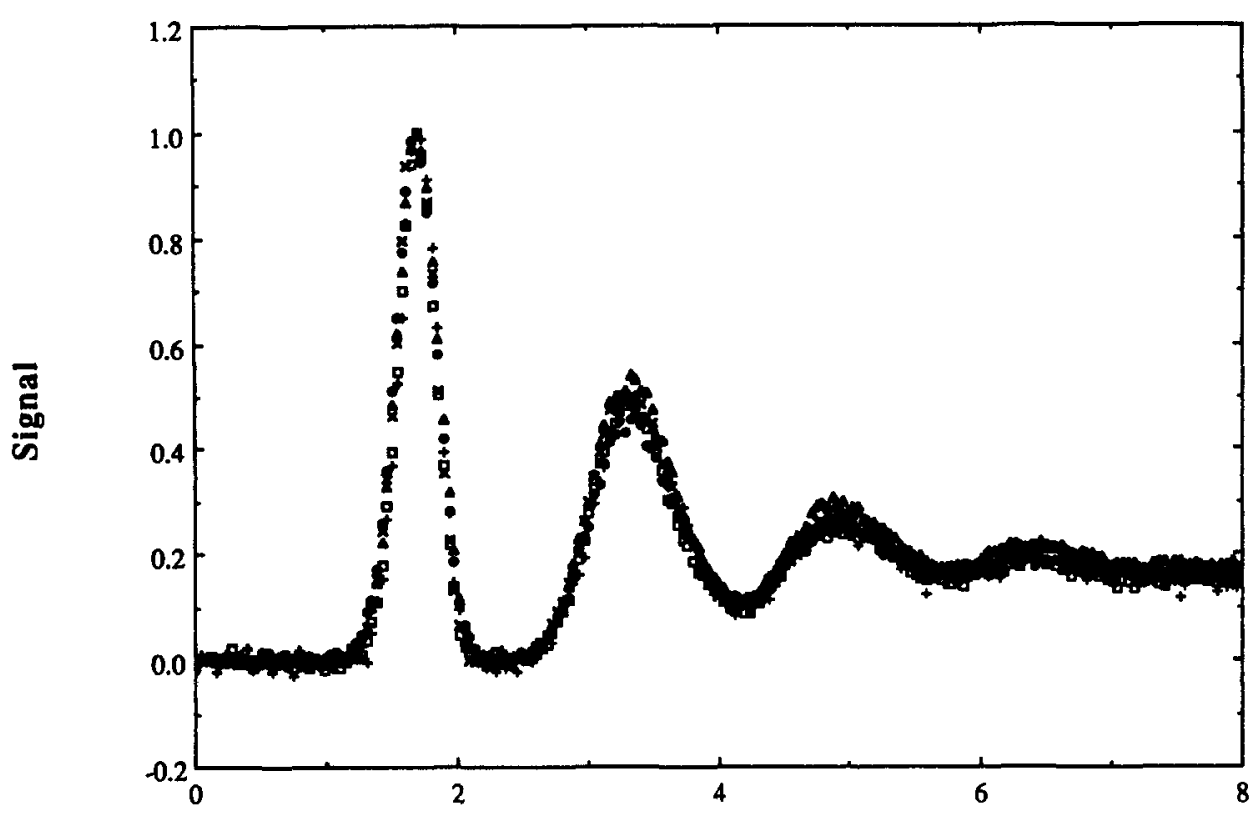

FIG. 17. Invariance of off-resonant FTS transient of $\mathrm{NaI}$ to pump and probe powers. $\lambda_{\text {pump }}=301 \mathrm{~nm}, \lambda_{\text {probe }}=620 \mathrm{~nm}$. Key to relative pump and probe powers: $\square$ for $P_{\text {pump }}=1.0, \quad P_{\text {probe }}=1.0 ; \times$ for $P_{\text {pump }}$ $=1.0, P_{\text {probe }}=0.21 ; \bullet$ for $P_{\text {pump }}=1.0$, $P_{\text {probe }}=22 ; \Delta$ for $P_{\text {pump }}=3.5, P_{\text {probe }}$ $=3.3 ;+$ for $P_{\text {pump }}=3.5, P_{\text {probe }}=0.12$. Each data set has been normalized to have unit height at the first peak. The zero of time has been arbitrarily chosen.

Time Delay (ps)

the on-resonant transient shape (not shown) was independent of the probe intensity.

In contrast to the off-resonance results, the on-resonant FTS transient changed dramatically as the pump power was increased, as illustrated in Fig. 18. When the pump is at low intensities, the transient has an initial fast rise followed by an ascending series of plateaus which washes out to a simple slow rise at longer times. The origin of this shape has been discussed in the previous section and is simply the "integral" of FTS transients for $\lambda_{2}^{*}$ probing. As the power of the pump is increased, however, the slower rising portion of the signal flattens out and for sufficiently high power even starts to descend. (Note also the ratio of the first rise to the asymptotic signal value.)

One possible explanation for the unexpected power dependence of the transient is that as the pump intensity is increased, a larger rotational-vibrational ensemble is excited. Hence, the preparation and dephasing of a rotationalvibrational wave packet becomes different. The rotational coherence phenomena has been observed in the reaction of $\mathrm{HgI}_{2}$ and $\mathrm{I}_{2},{ }^{40}$ and it is possible that as more and more levels are added to the packet by virtue of increasing the pump power, the dephasing rate becomes larger. This coherence may decay on a time scale of picoseconds. ${ }^{40,41}$ Also, the contribution of the $\Omega=1$ surface to the on-resonance signal at high powers may become significant. We are currently examining these points further.

\section{E. Pump bandwidth and pulse duration}

The effect of changing the bandwidth and pulse duration (or the uncertainty product) of the pump would be to prepare differently shaped wave packets on the excited state surface. It was of interest to us to see if an observable difference in the dynamics of the system could be detected. Figure
19 shows the results of two different pump bandwidths. (Note that for this particular experimental setup, both the pump and probe were derived from amplified CPM pulses. Consequently, the characteristics of the probe were changed as well as the pump. Changing the probe characteristics should not affect the dynamics.) In Fig. 19(a), the bandwidth of the $308 \mathrm{~nm}$ pump was $14 \mathrm{~nm}$ and the duration was $53 \mathrm{fs}$. In Fig. 19(b), the corresponding values were $1.5 \mathrm{~nm}$ and $225 \mathrm{fs}$, respectively. No effects other than temporal resolution changes were seen. It appears that the prepared wave packet is not much different, a point illustrated further by the theoretical calculations in the following section.

\section{THEORETICAL TREATMENT}

\section{A. Classical mechanical model}

For the dissociation reaction of ICN, close similarities were obtained in the results of the classical mechanical ${ }^{42}$ and quantum mechanical ${ }^{46}$ calculations. An interesting question is whether the alkali halide reaction can also be well modeled using a classical approach, similar to the one advanced by Bersohn and Zewail. ${ }^{42}$ A classical model may also provide insight into other important concerns regarding, for example, the dependence of FTS on the probe "window" and pump tuning. An additional, and more obvious, advantage lies in its comparative simplicity. Here, we develop the classical model used in our first publications, ${ }^{8,9}$ with emphasis on its application to the $\mathrm{NaI}$ reaction.

\section{Wave packet dynamics}

The potential energy surfaces were assumed to be identical to those used in Ref. 31. The ground (ionic) state ${ }^{47}$ $\left[V_{0}(R)\right]$ was discussed by Rittner, ${ }^{48}$ who considered a potential arising from a charge-charge interaction (giving a term with $R^{-1}$ dependence), and interacting dipoles (giving 


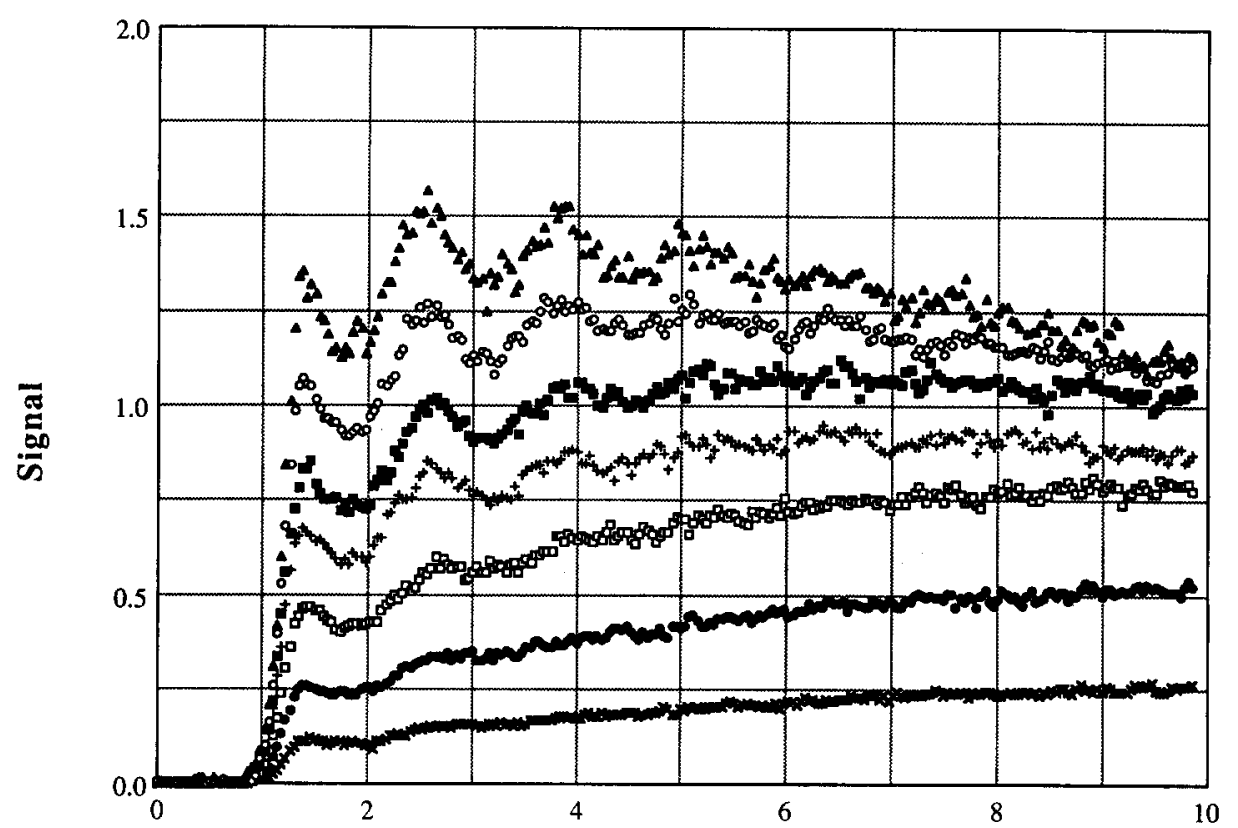

(a)

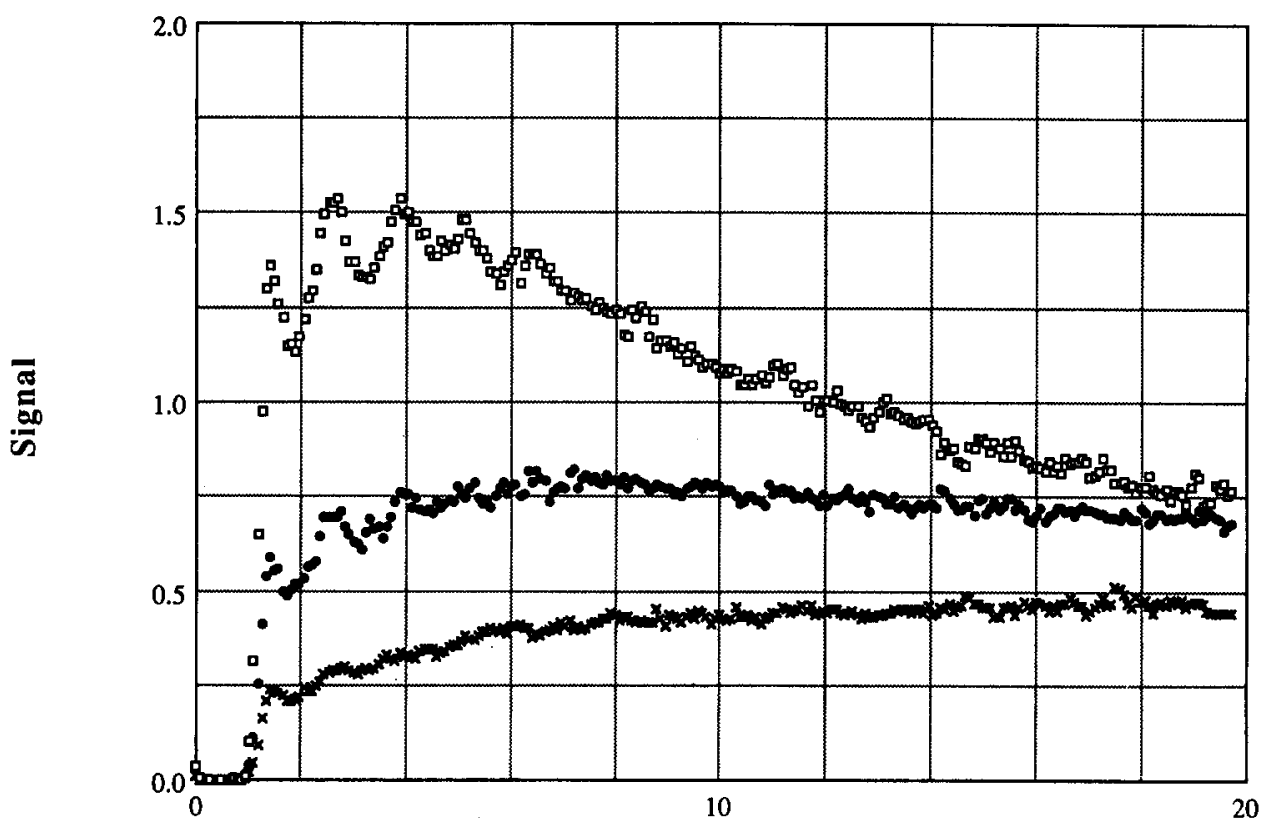

FIG. 18. Saturation of on-resonance FTS transient. (a) Initial absorption behavior as a function of pump power. Key for $P_{\text {pump }}$ (arbitrary units): $\times=0.1$; $\boldsymbol{O}=0.24 ; \square=0.49 ;+=0.91 ; \boldsymbol{\square}=1.6$; $\mathrm{O}=3.7 ; \boldsymbol{\Delta}=5.7$. At low powers, a series of plateaus are seen, but for higher powers, these features begin to resemble peaks. Note that the lowest power scans are increasing for $\tau>5 \mathrm{ps}$, whereas the higher power scans are either flat or decreasing, and also that the ratio of the first to second "step" changes with power. (b) Absorption behavior at long times, emphasizing the decay seen at high pump powers. Key for $P_{\text {pump }}$ (arbitrary units): $\times=0.36$; $\bullet=1.15 ; \square=5.5$.
$R^{-4}$ and $R^{-7}$ dependences). In addition, Rittner included an exponential repulsion term ( $e^{-R / \rho}$ dependence) and a van der Waals attraction ( $R^{-6}$ dependence). Faist and Levine $^{16}$ have suggested a slight modification of this Rittner potential so that the potential is well behaved at small $R$ (i.e., the potential must remain large and nonnegative as $R \rightarrow 0$ ) through the inclusion of an additional repulsive term (with $R^{-8}$ dependence) as a cofactor before the exponential repulsion. This new term is negligible at the internuclear separation of the well (or beyond). The explicit form of the potential is therefore given by ${ }^{16}$

$$
\begin{aligned}
V_{0}(R)= & \left\{A_{\text {ion }}+\left[\frac{B_{\text {ion }}}{R}\right]^{8}\right\} \exp \left[-\frac{R}{\rho_{\text {ion }}}\right]-\frac{e^{2}}{R} \\
& -\frac{e^{2}\left(\alpha_{\mathrm{M}^{+}}+\alpha_{\mathrm{X}^{-}}\right)}{2 R^{4}} \\
& -\frac{C_{\text {ion }}}{R^{6}}-\frac{2 e^{2} \alpha_{\mathrm{M}^{+}} \alpha_{\mathrm{X}^{-}}}{R^{7}}+E_{\mathrm{th}} .
\end{aligned}
$$

Here, $e$ is the charge of the electron, the $\alpha$ 's are the polarizabilities of the ions and $C_{\text {ion }}$ is the van der Waals coefficient. 


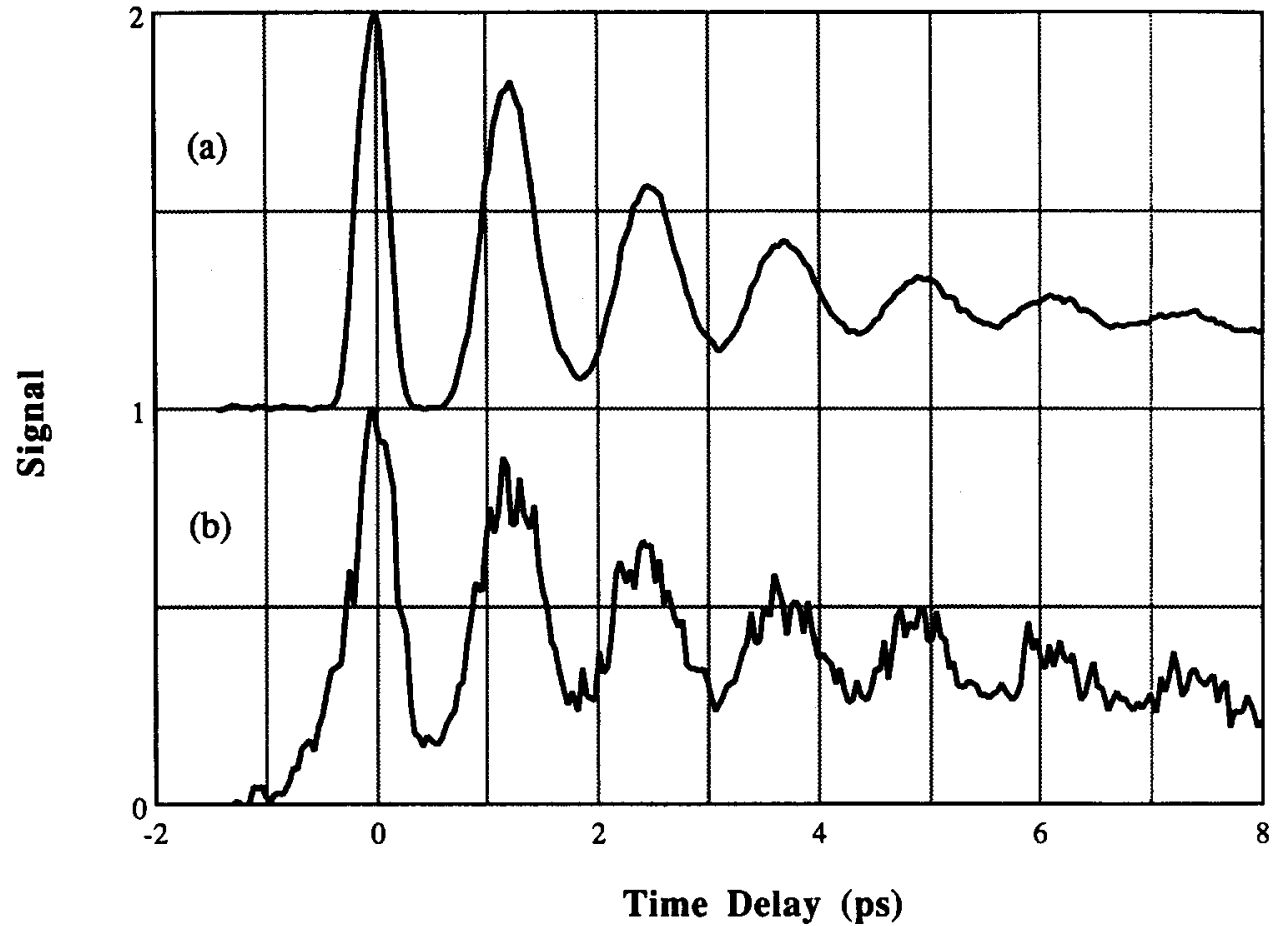

FIG. 19. Comparison of FTS off-resonant transient for $\mathrm{NaI}$ for different pulse widths. $\lambda_{\text {pump }}=308 \mathrm{~nm}$ and $\lambda_{\text {probe }}=616$ nm. (a) Results for temporally narrow pump and probe pulses, with spectral widths of 38.5 and $141 \AA$, respectively. (b) Results for temporally broad pump and probe pulses, with widths of 15 and 35 $\AA$, respectively. The shapes are basically similar; however, in case (b) the first peak is less well resolved.
The numerical values of the terms in this expression (as well as for the following two expressions) are given in Table $I$. The lowest-lying covalent state $\left[V_{1}(R)\right]$ was chosen (in accordance with van Veen $e t$ al.) to have a simple repulsive form $^{19(a)}$

$$
V_{1}(R)=A_{1} \exp \left[-B_{1}\left(R-R_{1}\right)\right] .
$$

In addition, an adiabatic coupling was assumed, of the form

$$
V_{01}(R)=A_{01} \exp \left[-\beta\left(R-R_{x}\right)^{2}\right] \text {. }
$$

The shape of the next higher lying potential surface $\left(V_{2}\right)$ is not well known. The most descriptive work on this PES of which we are aware is that of Bower et al. ${ }^{49}$ who made use of other available results. ${ }^{30,51}$ While the potential was found to be quite steep at short $R$, Bower et al. indicated

TABLE I. Values for the potential coefficients of Eqs. (6)-(8).

\begin{tabular}{lcl}
\hline \hline Coefficient & Value & Unit \\
\hline$A_{\text {ion }}$ & 2760 & $\mathrm{eV}$ \\
$B_{\text {ion }}$ & 2.398 & $\mathrm{eV}^{1 / 8} \AA$ \\
$C_{\text {ion }}$ & 11.3 & $\mathrm{eV}^{6}$ \\
$\rho_{\text {ion }}$ & 0.3489 & $\AA$ \\
$\alpha_{\mathrm{M}^{+}}$ & 0.408 & $\AA^{3}$ \\
$\alpha_{\mathrm{x}^{-}}$ & 6.431 & $\AA^{3}$ \\
$E_{\text {th }}$ & 2.075 & $\mathrm{eV}$ \\
$A_{1}$ & 0.813 & $\mathrm{eV}$ \\
$B_{1}$ & 4.088 & $\AA \AA^{-1}$ \\
$R_{1}$ & 2.7 & $\AA$ \\
$A_{01}$ & 0.055 & $\mathrm{eV}$ \\
$\beta$ & 0.6931 & $\AA \AA^{-2}$ \\
$R_{x}$ & 6.93 & $\AA$ \\
\hline \hline
\end{tabular}

$V_{2}(R)$ to be essentially flat for $R \geqslant 3 \AA$. (A small van der Waals well of a depth of $500 \mathrm{~cm}^{-1}$ is ignored here.) For simplicity, it has been assumed in the calculation that this PES is a constant over the range of internuclear separations explored by the reaction [ that is, $V_{2}(R)=V_{2}^{0}$ ]. The height of this constant potential surface is given by the energy of the $\mathrm{Na} D$-line absorption; these lines have a wavelength of about $589 \mathrm{~nm}$, corresponding to an energy of $V_{2}^{0}=17 \times 10^{3} \mathrm{~cm}^{-1}$.

Furthermore, we neglect the effects of rotational motion in the calculation. As shown by Engel et al. ${ }^{31}$ although the rotational energy is not entirely negligible at $650^{\circ} \mathrm{C}$, its effect on the calculated wave packet dynamics is relatively unimportant.

With these approximations, the scheme by which the expected LIF is calculated is straightforward. The spectral shape of the pump pulse is divided up into $N$ separate bins, each with its own characteristic wavelength $\left(\lambda_{i}\right)$ and amplitude $\left(a_{i}\right)$. Each bin is associated with a vertical transition from $V_{0}$ to $V_{1}$ at a unique internuclear separation near the bottom of the potential well of the ground state at $2.7 \AA$. (For the moment, we assume this transition to be instantaneous; the finite temporal pulsewidth of the pump will be considered later.) The initial conditions are taken to be

$$
R_{i}(t=0)=r_{i}
$$

and

$$
v_{i}(t=0)=0,
$$

where the $R_{i}(t)$ and $v_{i}(t)$ are the position and velocity, respectively, of the $i$ th particle at time $t$, and where $r_{i}$ is the solution of $V_{1}(R)-V_{0}\left(R_{0}\right)=h c / \lambda_{i}$.

We now consider the motion on the adiabatic potential well for $t>0$. The classical equations of motion in the center of mass frame are given by 


$$
\begin{aligned}
F_{i}=\mu a & =\mu \frac{d v_{i}}{d t} \\
& =-\frac{d V\left(R_{i}\right)}{d R_{i}},
\end{aligned}
$$

where $\mu$ is the reduced mass. Here, $V\left(R_{i}\right)$ is the shape of the adiabatic potential well which can be obtained by diagonalizing the $2 \times 2$ matrix involving $V_{0}, V_{1}$ and $V_{01}$. Since $V_{01}$ is relatively small,

$$
V(R) \simeq V_{j}(R)+V_{01}(R),
$$

where $j=0$ for $R_{i} \geqslant R_{x}$ and $j=1$ for $R<R_{x} . R_{x}$ is the separation at which the two surfaces cross, and is approximately equal to $7 \AA$. Near $R_{x}$, Eq. (13) is an excellent approximation and is valid at all $R$ 's since $V_{01}$ falls off sufficiently rapidly with increasing $R$. Equation (12) above can be expressed as a system of first-order differential equations:

$$
\begin{aligned}
& \frac{d R_{i}}{d t}=v_{i}, \\
& \frac{d v_{i}}{d t}=-\frac{1}{\mu} \frac{d V\left(R_{i}\right)}{d R} .
\end{aligned}
$$

These equations were numerically integrated, using a fourth-order Runge-Kutta algorithm, ${ }^{52}$ yielding the positions and velocities of all of the particles at the times $t=0$, $\Delta t, 2 \Delta t, \ldots, M \Delta t$ for given $\Delta t$ and $M$.

The probability for an interlevel crossing of a wave packet is given by Eq. (6). In calculating the off-resonant signal, the level crossing was therefore treated as follows: The amplitude $a_{i}$ of each particle $i$ was reduced by a multiplicative factor each time it encountered the level crossing. The magnitude of this factor was determined from Eq. (6). These reductions in the particle amplitudes were accumulated together in a separate sum, representing the relative fraction of molecules which had escaped the well, and was used in estimating the on-resonant signal.

Note that the probability for wave packet crossing is independent of the direction of travel. However, when the velocity of the packet is negative, the transition (if it occurs) will be from the excited surface back to the ground state well. These molecules have effectively escaped the adiabatic trapping and (after a single pass on the ground state surface) dissociate. However, a small fraction will return to the adiabatic well by a second crossing after a complete round trip; these molecules may lead to a rising background probe absorption. Because of the complexity of the dynamics, this pathway is ignored in the classical model.

The off-resonant signal is determined by summing together the absorptions of each of the particles, taking (for example) a Lorentzian line shape ${ }^{42}$ :

$$
S^{\text {off }}(t)=\sum_{i=1}^{\mathrm{N}} \frac{a_{i}}{1+\left\{\left[\Delta E_{i}(t)\right] / \gamma\right\}^{2}},
$$

where

$$
\Delta E_{i}(t) \equiv V_{2}^{0}-\left\{V\left[R_{i}(t)\right]+h \omega\right\}
$$

and where $\omega$ and $\gamma$ are the central frequency and halfwidth corresponding to the probe spectrum. Note that we have implicitly assumed in Eq. (15) that natural linewidth for the absorption is negligible relative to spectral width of the probe. For the opposite case, the extension is straightforward.

We will generally follow the approximation made by Engel et al..$^{31}$ that the magnitude of the transition dipole for the absorption is a constant with respect to $R$. This is the case of the Condon approximation. Since the nature of the bond changes (i.,e., ionic vs covalent) as the molecule moves in the adiabatic well, this approximation may be invalid over certain ranges. Therefore, we will also consider the effects of relaxing this assumption. In this case, Eq. (15) is replaced by

$$
S^{\text {off }}(t)=\sum_{i=1}^{\mathrm{N}} \frac{a_{i} F\left[R_{i}(t)\right]}{1+\left\{\left[\Delta E_{i}(t)\right] / \gamma\right\}^{2}},
$$

where $F\left[R_{i}(t)\right]$ is a multiplicative factor depending on $R_{i}(t)$, the instantaneous position of the $i$ th particle. For example, the transition cross section might drop substantially on the right-hand side on the adiabatic well, where the nature of the bond is primarily ionic. This effect can be most crudely modeled by taking $F$ to be a step function:

$$
\begin{aligned}
& F(R)=1, \text { for } R<R_{x} \\
& =0 \text {, otherwise. }
\end{aligned}
$$

Finally, it is necessary to take into account the finite pulsewidths of both the pump and the probe sources. This was accomplished by numerically convolving the results of Eq. (17) [or Eq. (15)] with an assumed response function characterizing the experiment, which was taken to be a Gaussian in shape. ${ }^{42}$

\section{Results of the classical model}

Calculations were performed in order to simulate the experimental results over a wide range of parameters. Typical simulation results for both the on- and off-resonance cases are shown in Fig. 20. Studies were made of the effects of both pump and probe tuning. A variety of other issues giving insight into the dynamics of the reaction have also been considered.

For example, we must now resolve the issue of precisely where in the well the probe absorption takes place. For probe wavelengths to the red of $589 \mathrm{~nm}$, a vertical transition will generally be allowed at two distinct ranges of separation (i.e., optically coupled regions or OCR's) on either side of the well bottom at $R_{x}$. In Fig. 21, which is also illustrative of the probe tuning results, the simulations are shown for the experiment at two separate probe wavelengths for two choices of the multiplicative factor $F(R)$, defined above. The first choice assumes a constant transition dipole across the well $[F(R)=1$ for all $R]$, so that the two OCR's each contribute to the observed signal. The second choice, as given in Eq. (18) above, presumes that the region occurring at $R>R_{x}$ has a negligible transition dipole.

It is apparent from Fig. 21 that the alternative of a constant value of $F(R)$ can be ruled out. For probe wavelengths sampling the bottom of the well [E.g., $\lambda_{\text {probe }}=620 \mathrm{~nm}$, as in Fig. 21 (a) ] the peaks are expected to be equally spaced. This is only because of the limited temporal resolution; the unconvolved data shows there to be two separate peaks slightly separated. As the probe is tuned so that the absorption is 


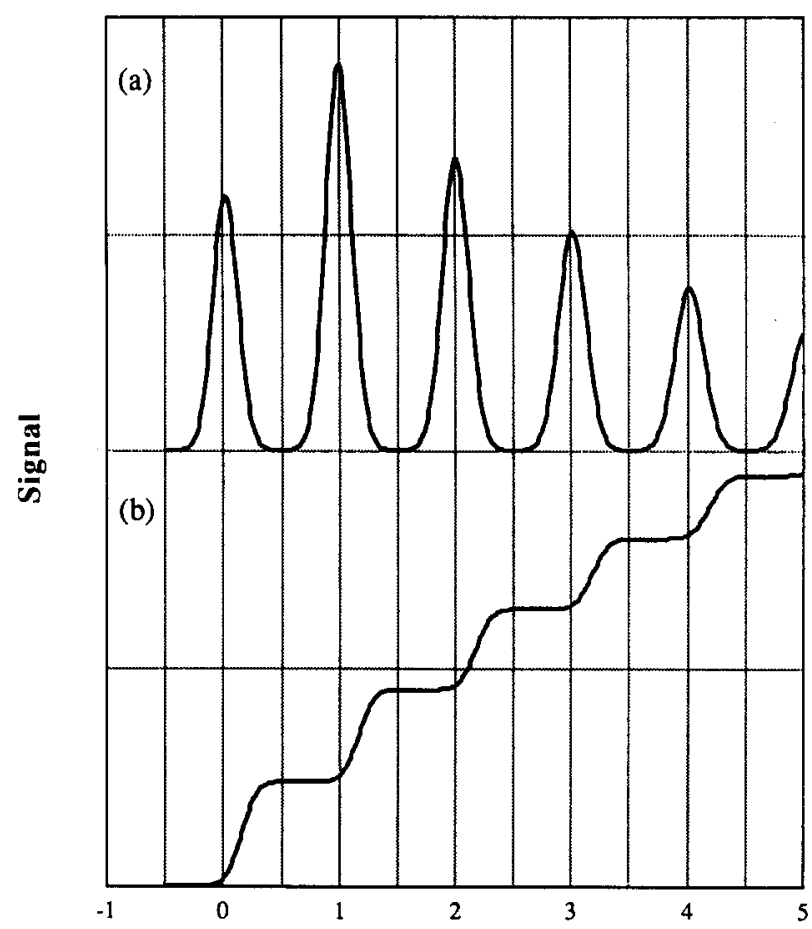

Time Delay (ps)

FIG. 20 Typical results for $\mathrm{NaI}$ transients obtained from the classical model simulation. Key: (a) off-resonant transient $\left(\lambda_{\text {probe }}=620 \mathrm{~nm}\right)$; (b) on-resonant transient $\left(\lambda_{\text {probe }}=589 \mathrm{~nm}\right.$ ). The pump wavelength is taken to be 324 $\mathrm{nm}$ with a width of $3.0 \mathrm{~nm}$. The convolution used in the calculation assumes a 150 fs temporal resolution.

away from the bottom of the well, the separation of these peaks in the simulated absorption grows. For $\lambda_{\text {probe }}=720$ nm [Fig. 21(c)], the two peaks are resolved, so that the temporal separation between peaks is no longer a constant. This is in direct contradiction with the experimental transients, where the absorption peaks for each transient were uniformly spaced for data obtained at every probe wavelength. In all subsequent calculations, we have therefore taken the multiplicative factor $F(R)$ to be a step function [Figs. 21 (b) and 21(d) ].

Furthermore, Fig. 21 illustrates that except for a constant scaling factor, the results obtained for the two probe wavelengths are virtually identical. This is consistent with the experimental results presented above, where no large dependence of the transient on probe wavelength was observed (for wavelengths away from the free-Na transition).

Perhaps the most successful aspect of the classical model is its ability to correctly predict the peak frequency as a function of the pump wavelength remarkably closely to the experimental results. Figure 22 shows the results of the calculation for a number of pump wavelengths. The calculated and experimentally measured periods are listed in Table II; the agreement is clearly very good. Such close agreement circumstantially supports the assumed potential energy surfaces, Eqs. (7) and (8), with no adjustable parameters.

The shape of the calculated absorptions (see, for example, Fig. 20) differ from the observed transients in two im-

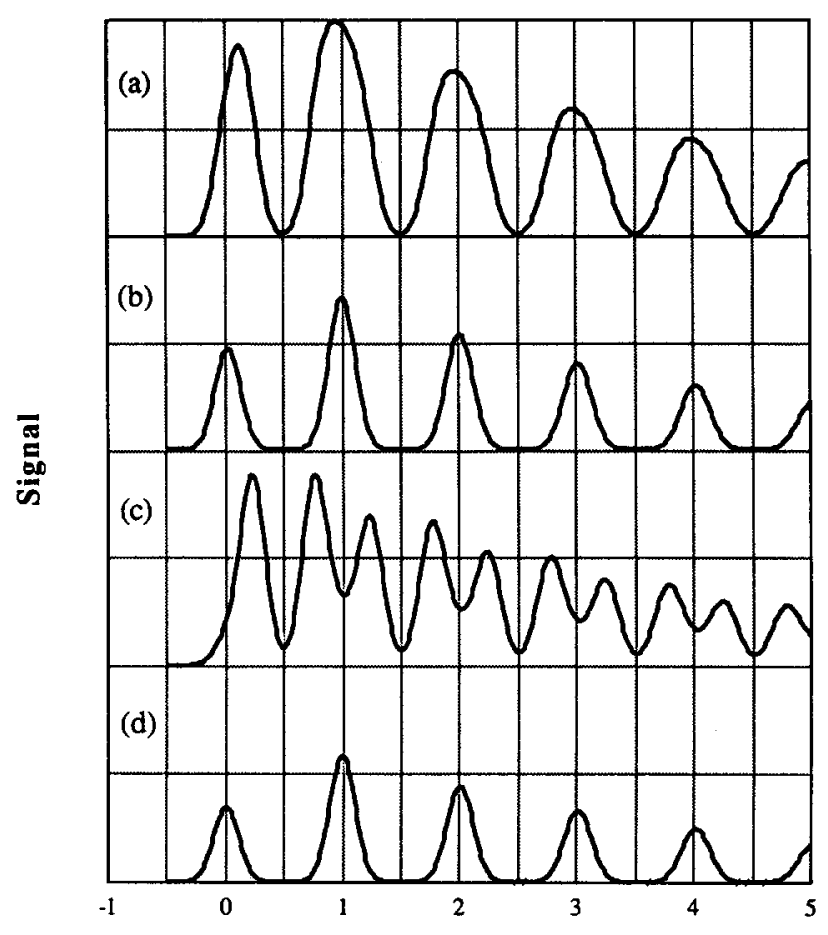

Time Delay (ps)

FIG. 21. Simulation results for different probe wavelengths and choices of $F(R)$. Key: (a) $\lambda_{\text {probe }}=620 \mathrm{~nm}$ and $F(R)=1$ for all $R$ (the constant dipole limit); (b) $\lambda_{\text {probe }}=620 \mathrm{~nm}$ and $F(R)=1$ for $R<R_{x}$ and $F(R)=0$ otherwise (the step-function dipole limit); (c) $\lambda_{\text {probe }}=720 \mathrm{~nm}$ and constant dipole limit; and (d) $\lambda_{\text {probe }}=720 \mathrm{~nm}$ and step-function dipole limit. The constant dipole cases, particularly (c), clearly does not resemble the experimental data (see the text). Note the similarity of the step-fuunction traces, (b) and (d), showing the independence in this limit of the transient shape to the probe wavelength.

portant respects. First, the height of the first peak, perhaps unexpectedly, is less than that of the second peak. Insight into this behavior can be gathered from Fig. 23, which explicitly shows the motion of the wave packet on the upper state well. After it is prepared by the pump (assumed instantaneous for this calculation), the wave packet rapidly gains kinetic energy, passes through the OCR and continues on to the ionic part of the surface $\left(R>R_{x}\right)$. Because the slope of the ionic potential is relatively small compared to the FrankCondon part, the packet spends proportionally much more time there. After $\sim 1 \mathrm{ps}$, the wave packet returns to the Frank-Condon region, where two passes of the OCR (one in each direction) are made in very rapid succession (within about $60 \mathrm{fs}$ ). When the finite width of the pump and probe pulses are included into the calculation (via convolution), these separate peaks are no longer resolved. The small height of the first peak is, therefore, explained because a single pass of the OCR is involved, whereas the subsequent peaks result from a double pass.

The apparent discrepancy between the calculation and the experimental data, where the first peak was generally observed to be greater in height than the subsequent peaks, may be explained. Only the $\Omega=0^{+}$pathway is adiabatic in nature; molecules in the $\Omega=1$ state promptly dissociate, 


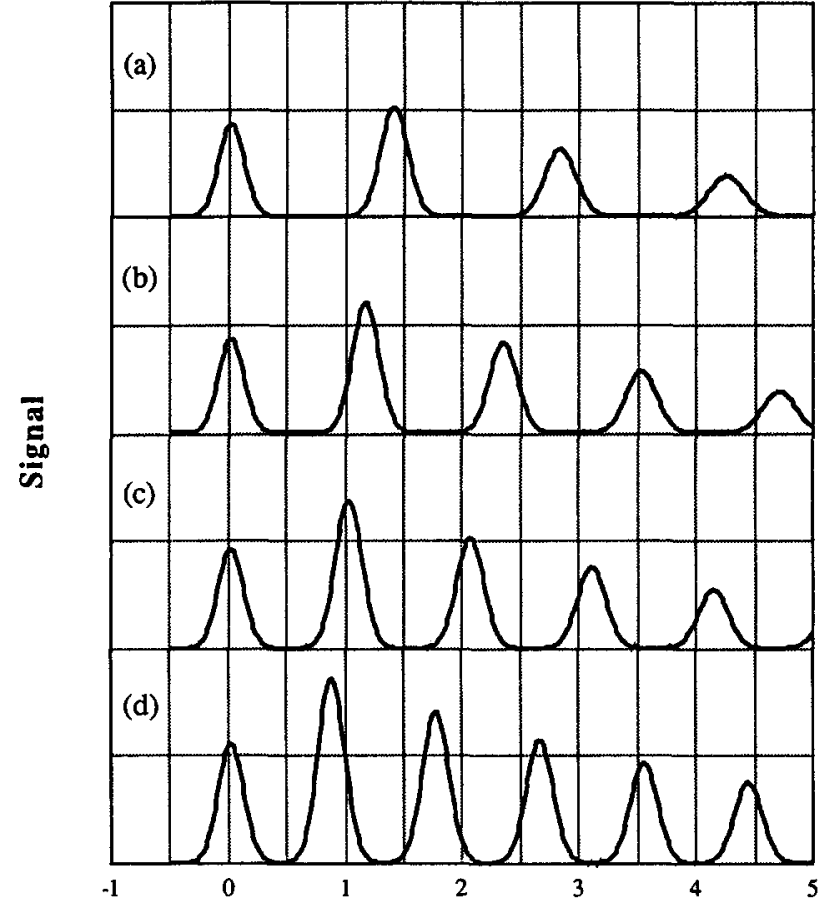

Time Delay (ps)

FIG. 22. Simulation results for several pump wavelengths. Key (a) $\lambda_{\text {nump }}$ $=300 \mathrm{~nm}$; (b) $\lambda_{\text {pump }}=311 \mathrm{~nm}$; (c) $\lambda_{\text {pump }}=321 \mathrm{~nm}$; and (d) $\lambda_{\text {pump }}$ $=339 \mathrm{~nm}$. Comparison with the experimental results for Fig. 12 shows good agreement.

contributing only to the first peak (see also Sec. III B 2). The calculation, however, does not take into account the latter pathway.

A more significant difficulty is the failure of the model to accurately predict the dispersion of the packet within the well. Such dispersion is evident in the experimental results by the increase in width of the second and subsequent peaks relative to that of the first peak and eventually by the rising baseline. Using the experimentally appropriate parameters in the simulation, the model predicts a negligible amount of dispersion.

We postulate that the error with the calculation in this regard lies in the extremely restricted range of the initial separations, for which the full width at half-maximum (FWHM) is typically only $\Delta R=0.01 \AA$. In order to investigate the effect of a broader range of such initial separations $\Delta R$, the calculations were repeated with different choices in

TABLE II. Measured and calculated values for the wave packet period. The measured values were obtained directly from the raw data. The calculated values were determined from the classical model described in the text.

\begin{tabular}{ccc}
\hline $\begin{array}{c}\text { Wavelength } \\
\text { (nm) }\end{array}$ & $\begin{array}{c}\text { Experimental } \\
\text { period (ps) }\end{array}$ & $\begin{array}{c}\text { Calculated } \\
\text { period (ps) }\end{array}$ \\
\hline 300 & 1.64 & 1.412 \\
311 & 1.20 & 1.173 \\
321 & 1.09 & 1.031 \\
339 & 0.98 & 0.885 \\
\hline
\end{tabular}

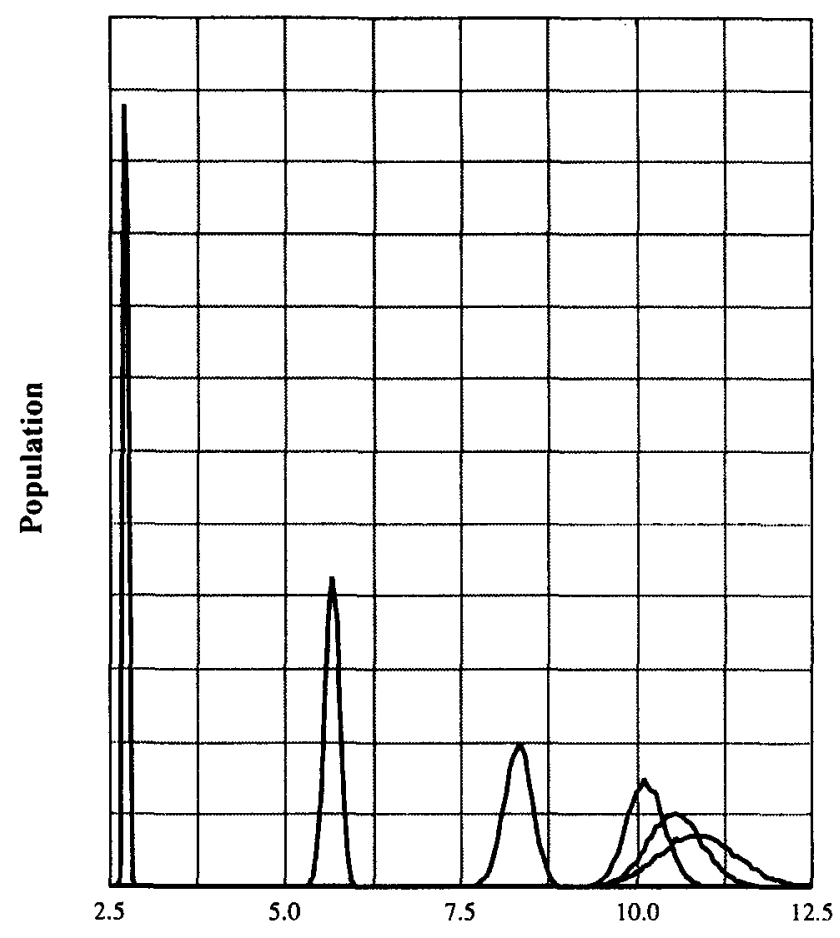

Separation ( $\AA$ )

FIG. 23. Simulation of the spatial evolution of the wave packet within the excited state well. From left to right, the peaks represent the wave packet at time $t=0$ (immediately after the pump pulse, assumed here to be instantaneous), $t=100 \mathrm{fs}, t=200 \mathrm{fs}, t=300 \mathrm{fs}, t=400 \mathrm{fs}$, and $t=500 \mathrm{fs}$. Subsequently, the packet, which has reached its extreme travel at $t=500 \mathrm{fs}$, retraces these steps back to short $R$ (however, the broadening of the packet by dispersion continues to develop). Since the crossing occurs at $R \sim 7 \AA$, the packet clearly spends most of the time on the ionic surface.

the spectral width of the pump. As shown in Fig. 24, an increase in this parameter by a factor of about 4 from its nominal value of $30 \AA \mathrm{FWHM}$ was found to give much more satisfactory results. Recall that the packet considered here is the "instantaneous" packet (without taking into account the temporal resolution of the pulses used). As discussed above

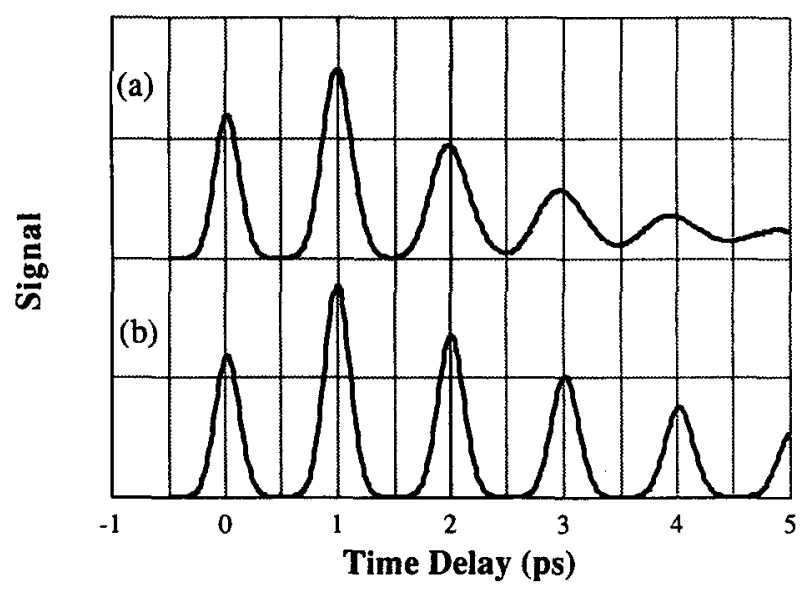

FIG. 24. Simulation results as a function of the spectral width of the pump pulse. Key: (a) $\Delta \lambda_{\text {pump }}=15.0 \mathrm{~nm}$ (corresponding to an initial range of separation of $\Delta R=0.05 \AA$ ); and (b) $\Delta \lambda_{\text {pump }}=3.0 \mathrm{~nm}$ (corresponding to an initial range of separation of $\Delta R=0.01 \AA$ ). 
and elsewhere, ${ }^{42}$ this temporal resolution is taken into consideration in the final convolution with the pulse shape. In the quantum calculation (next section), however, a "train of packets" is built-in as part of the initial preparation.

Finally, the effects of relaxing the condition of Eq. (11), where the velocities of the initially prepared states are all assumed to be zero, have been considered. This approximation is not entirely unjustified, since the vibrational energy of the ground-state molecule is fairly small in comparison to kinetic energy of the fragments at the terminal velocity of recoil.

\section{B. Quantum mechanical and semiclassical models}

A quantum mechanical model of the dynamics of an $\mathrm{NaI}$ molecule after excitation by a femtosecond pump pulse has previously been developed by Engel et $a l^{31}$ In this approach, the dissociation was treated by considering the evolution of a wave packet on the appropriate potential energy surface. The calculation is performed by numerical integration of the time-dependent Schrödinger equation. Since the shapes of the potential surfaces were assumed, the quantum calculation was performed without adjustable parameters.

The results obtained by Engel et al. (Fig. 25) are similar to our experimental results in a number of respects. The general shapes of the transients both on resonance and off resonance have the correct form (i.e., rising plateaus and a series of equally spaced, decaying peaks, respectively). Further, the oscillation frequency matches fairly well to the data, and shows the correct pump wavelength dependence. The offresonant signal calculated by Engel et al. (as shown in Fig. 25 ) is proportional to the fraction of excited molecules in the covalent state whose interatomic distance is less than $R_{x}$, the crossing point of the two surfaces. This presupposes that the optically coupled region for the probe absorption is the entire range $R<R_{x}$. It is interesting to note that the quantum calculations, as well as the classical results, do not reproduce the experimentally observed rapid decay of the peak amplitudes.

Quantum calculations have also been performed by Choi and Light, ${ }^{32}$ using the Gauss-Chebyshev discrete variable representation. ${ }^{53}$ The propagation of the wave packet in

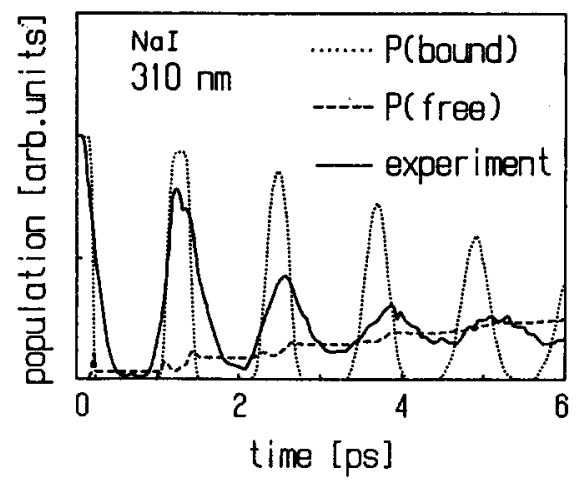

FIG. 25. Results of quantum simulation of the experiment. The bound and free neutral populations and the experimental results for $\mathrm{NaI}$ are shown. The pump pulse is taken to be at $310 \mathrm{~nm}$ with a full width of $100 \mathrm{fs}$. Taken from Ref. 31 . the framework of the general time-dependent theory of Hell$\mathrm{er}^{54}$ was implemented under the influence of the Hamiltonian for the interacting diabatic states. using the full quantum technique developed by Light,${ }^{53}$ the time-dependent Schrodinger Equation was solved for the motion on the upper state (adiabatic) surface. The evolving wave packet was found by Choi and Light to oscillate with a period of approximately $1 \mathrm{ps,}$, in agreement with our experimental results.

Another approach is that of Marcus, ${ }^{33}$ who utilized a semiclassical angle variable representation, which previously had been developed in connection with the theory of molecular collisions. The evolution of the wave packet generated from a femtosecond Gaussian pulse is considered in both a one-dimensional and multidimensional case. This technique reproduces the main results of the quantum calculation of Engel et al..$^{31}$ Simple expressions were obtained, and the approach may allow for calculations involving multidimensional systems.

Most recently, Lin and Fain ${ }^{34}$ have independently developed a theoretical approach to the FTS technique and applied their results to the NaI case. Their method is based on application of the Liouville equation to the appropriate density matrix for the system. It was found that for a particular choice of physical parameters, the off-resonant FTS results could be approximately reproduced, in that oscillations were similar to the experimentally observed frequency. The derived form for the transient shape was $\exp (-R t)[1+b$ $X \cos \omega t]$. The exponential term represents the decaying envelope due to level crossing depletion and the second term describes the oscillation of the packet in the well. Using $\omega=0.8 \times 10^{12} \mathrm{~s}^{-1}, R=0.8 \times 10^{11} \mathrm{~s}^{-1}$, and $b=0.9$, they reproduced the general shape of the FTS of our experiments.

\section{OTHER ALKALI HALIDES}

The alkali halides, $\mathrm{NaBr}$ and $\mathrm{LiI}$, were chosen for comparison with $\mathrm{NaI}$ because the former was expected to behave diabatically, while the latter was predicted to behave similarly to $\mathrm{NaI} .^{12,15,19}$ The $\mathrm{NaBr}$ should not exhibit major trapping, and hence no oscillations (or possibly only weak oscillation) should occur in the FTS signal. The LiI would be expected to produce oscillations, but at a higher frequency.

In Fig. 26(a) and 26(b), the data for on- and off-resonance $\mathrm{NaBr}$ experiments are respectively displayed. For both cases, the $310 \mathrm{~nm}$ pump was derived from the second harmonic of the PDA. The $625 \mathrm{~nm}$ off-resonant probe [Fig. 26(a)] and $589 \mathrm{~nm}$ on-resonant probe [Fig. 26(b)] were obtained by continuum generation. On resonance, the $\mathrm{NaBr}$ signal appears similar to that of $\mathrm{NaI}$ except that it possesses a noticeable overshoot near $t=0$ and that there are no apparent oscillations. The overshoot probably results from the offresonance contribution signal owing to the large bandwidth of the probe. The contribution of the off-resonance signal to the on-resonance signal has been observed in $\mathrm{NaI}$ as well and has been discussed in Sec. III. The absence of oscillations is confirmed by the off-resonance signal which exhibits severe damping such that only a faint recurrence is observed after the initial peak. $\mathrm{NaBr}$ is predicted to have a Landau-Zener parameter at least an order of magnitude larger than that of 


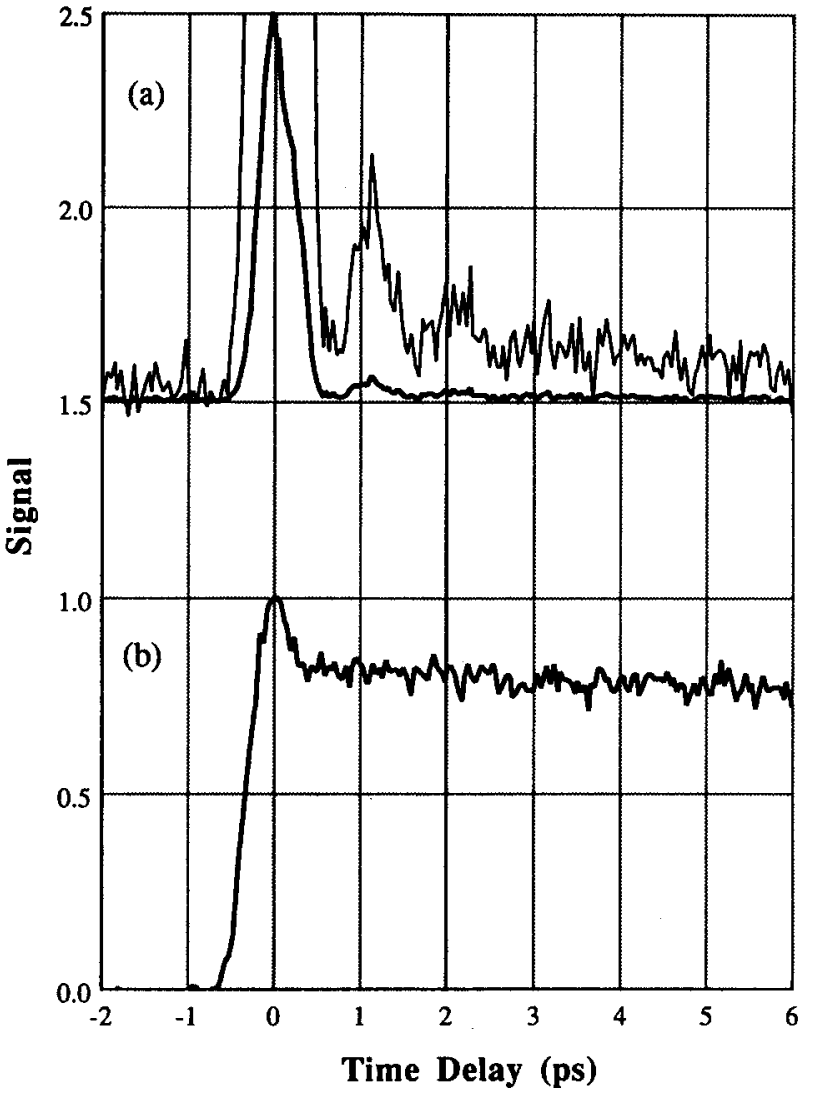

FIG. 26. FTS transients for $\mathrm{NaBr}$. (a) Off-resonance $\left(\lambda_{\text {probe }}=627 \mathrm{~nm}\right)$; (b) on-resonance $\left(\lambda_{\text {probe }}=590 \mathrm{~nm}\right)$. For case (a), the data is also shown (thin line) in an expanded scale (times ten) in order to emphasize the secondary peaks. (See the text.)

$\mathrm{NaI}$; therefore, it should behave diabatically. The excited and ground state $\mathrm{NaBr}$ PES effectively cross allowing for the escape of the $\mathrm{Na}$ and $\mathrm{Br}$ fragments after only one pass through the critical region. The small second peak in the offresonant data indicates that a very small percentage of the fragments are trapped. It was also noted that the period of the $\mathrm{NaBr}$ oscillation was close to that of the $\mathrm{NaI}$ for the pump wavelength of $310 \mathrm{~nm}$. $^{55}$

The experimental conditions for the LiI experiments were similar to that of NaI. For the on-resonance data, the probe was tuned to the $2^{1 / 2} P_{3 / 2,1 / 2} \leftarrow 2^{1 / 2} S_{1 / 2}$ transitions (at 6709.6 and $6709.8 \AA$ ); the $310 \mathrm{~nm}$ pump was derived from SHG of the PDA. The on-resonance data is typical of that seen for $\mathrm{NaI}$ and $\mathrm{NaBr}$, except that the initial overshoot is much larger. Again, because of the large probe bandwidth, the off-resonance signal contributes to the on-resonance data. When the probe is tuned off resonance to the blue (e.g., to $660 \mathrm{~nm}$ ), an oscillation becomes visible and the relative height of the first peak increases rather substantially. This scan has a very similar appearance to the $\mathrm{NaI}$ data taken at very red probe wavelengths except that the modulation depth is very small compared to that observed in $\mathrm{NaI}$; the period is also smaller than that of $\mathrm{NaI}$.

In contrast to the blue results, off-resonance tuning to the red did not yield multiple absorption peaks, although the overall shape of the transient is otherwise similar. This result is different from that of both $\mathrm{NaI}$ and $\mathrm{NaBr}$. The results for $\mathrm{LiI}$ and $\mathrm{NaBr}$ should be considered preliminary as we must complete the study and characterize the effect of varying the pump and the probe wavelengths on the observed FTS transients.

\section{CONCLUSIONS}

In this paper, femtosecond transition-state spectroscopy (FTS) was employed to interrogate the dissociation reactions of alkali halides in real time. Results for the reaction of $\mathrm{NaI}$ were presented which allowed for the characterization of the dynamics. The appearance of an oscillation (resonance) in the FTS results indicated that some NaI molecules were being trapped in a well formed by the avoided crossing of the covalent and ionic PES. The time between the peaks yielded the traversal time of the molecule (wave packet) across the well. The damping gave the probability of escape out of the well (and hence the coupling of the surfaces).

The influence of various experimental parameters on the NaI dynamics was evaluated in detail. The two most notable points were the effect of the total available energy (pump wavelength) and power on the FTS transients. Tuning of the pump resulted in the variation of the oscillation period of the signal which allowed for the measurement of the potential well degree of anharmonicity. Furthermore, the relative contribution of the other dissociation pathways, which did not lead to trapping of the photofragments, was also discussed.

In addition to the $\mathrm{NaI}$ reaction, the dissociation of $\mathrm{NaBr}$ and LiI was studied and preliminary results were presented. The former, which was predicted to behave diabatically, did not exhibit strong trapping. On the other hand, the fragments from the LiI dissociation were trapped; however, the modulation depth of the oscillation was considerably less than that observed in NaI. Further studies of these molecules involving the tuning of the pump wavelength are required for a full comparison with NaI.

The FTS results presented here are reproduced using classical mechanics, as we invoked in our earlier reports. ${ }^{8,9}$ The results are also obtained from quantum mechanical calculations $^{31,32,34}$ and from semiclassical methods. ${ }^{33}$ It may turn out that the experiments and theories on alkali halide reactions are prototype for investigating real-time femtosecond reaction dynamics, and for "seeing" wave packet motion in elementary reactions. This is reminiscent of the "alkali age" era of molecular beams some 30 years ago.

Note added in proof: In a recent article by Lee et al. ${ }^{57}$ the classical mechanical FTS model of Ref. 42 was extended to $\mathrm{NaI}$ dissociation. They obtained the general oscillatory behavior and also deduced the observed period. Our classical mechanical model results are detailed in the text.

\section{ACKNOWLEDGMENTS}

This work was supported by Air Force Office of Scientific Research. Contribution No. 7966. We wish to thank Martin Gruebele for his helpful comments on the manuscript. 


\section{APPENDIX}

We consider the frequency of a wave packet traveling on a Morse potential, which has the form

$$
V(R)=D_{e}\left\{1-\exp \left[-a\left(R-R_{e}\right)\right]\right\}^{2} .
$$

The vibrational energy spacing for this potential is given by the relation

$$
E_{n}=h v_{0}(n+1 / 2)-\chi h v_{0}(n+1 / 2)^{2},
$$

where $v_{0}$ is the harmonic frequency and $\chi$ is the anharmonic constant. This energy separation determines the oscillation frequency of a wave packet made up of a superposition of vibrational states, according to the relation ${ }^{56}$

$$
\begin{aligned}
v_{\text {packet }} & =\frac{1}{h} \frac{\partial E}{\partial n} \\
& =v_{0}(1-2 n \chi-\chi) .
\end{aligned}
$$

If it is assumed that the transition to the adiabatic surface takes place from very nearly the bottom of the ground state well (i.e., neglecting rotational and vibrational energy), then the packet energy is

$$
E_{\text {packet }}=h\left(v_{\text {pump }}-v_{\text {diss }}\right) \text {. }
$$

Here, $v_{\mathrm{pump}}$ and $v_{\text {diss }}$ are the frequencies corresponding to the pump photon and dissociation energies, respectively. Applying Eq. (A2), the packet energy becomes

$$
v_{\text {pump }}-v_{\text {diss }}=v_{0}(n+1 / 2)-\chi v_{0}(n+1 / 2)^{2} \text {. }
$$

Next, we solve for $n$. From Eq. (A4),

$$
n=\frac{1-\chi-\left(v_{\text {packet }} / v_{0}\right)}{2 \chi},
$$

so that

$$
n+\frac{1}{2}=\frac{1-\left(v_{\text {packet }} / v_{0}\right)}{2 \chi}
$$

Substituting this result into Eq. (A6) above yields

$$
v_{\text {pump }}-v_{\text {diss }}=\frac{v_{0}}{4 \chi}-\frac{v_{\text {packet }}^{2}}{4 \chi v_{0}} \text {. }
$$

This expression can be arranged into a somewhat simpler form

$$
v_{\text {packet }}^{2}=v_{0}^{2}-4 \chi v_{0}\left(v_{\text {pump }}-v_{\text {diss }}\right) .
$$

${ }^{1}$ (a) M. J. Rosker, M. Dantus, and A. H. Zewail, J. Chem. Phys. 89, 6113 (1988); (b) M. Dantus, M. J. Rosker, and A. H. Zewail, ibid. 89, 6128 (1988); (c) R. B. Bernstein and A. H. Zewail, ibid. 90, 829 (1989).

${ }^{2}$ N. F. Scherer, J. L. Knee, D. D. Smith, and A. H. Zewail, J. Phys. Chem. 89, 5141 (1985)

${ }^{3}$ N. F. Scherer, L. R. Khundkar, R. B. Bernstein, and A. H. Zewail, J. Chem. Phys. 87, 1451 ( 1987).

${ }^{4}$ M. Dantus, M. J. Rosker, and A. H. Zewail, J. Chem. Phys. 87, 2395 (1987).

${ }^{5}$ M. J. Rosker, M. Dantus, and A. H. Zewail, Science 241, 1200 (1988).

${ }^{6}$ R. M. Bowman, M. Dantus, and A. H. Zewail, Chem. Phys. Lett. 156, 131 (1989).

${ }^{7}$ For a review, see: (a) A. H. Zewail, Science 241, 1645 (1988); (b) A. H. Zewail and R. B. Bernstein, Chem. Eng. News 66, 24 (1988).

${ }^{8}$ T. S. Rose, M. J. Rosker, and A. H. Zewail, J. Chem. Phys. 88, 6672 (1988).

${ }^{9}$ M. J. Rosker, T. S. Rose, and A. H. Zewail, Chem. Phys. Lett. 146, 175 (1988).
${ }^{10}$ M. Dantus, R. Bowman, M. Gruebele, and A. H. Zewail, J. Chem. Phys. 91, 7437 (1989).

"D. R. Herschbach, Angew. Chem. Int. Ed. Engl. 26, 1221 (1987).

${ }^{12}$ R. S. Berry, in Alkali Halide Vapors, edited by P. Davidovits and D. L. McFadden (Academic, New York, 1979) and references therein for earlier work.

${ }^{13}$ (a) L. D. Landau, Phys. Soviet Union 2, 46 (1932); (b) C. Zener, Proc. R. Soc. London Ser. A 137, 696 (1932); (c) R. D. Levine and R. B. Bernstein, Molecular Reaction Dynamics and Chemical Reactivity (Oxford University Press, New York, 1987).

${ }^{14}$ Reference 12 gives a number of articles on the subject and Refs 15-21 give examples of work in different areas of alkali halide studies, especially those more recent.

${ }^{15}$ R. Grice and D. R. Herschbach, Mol. Phys. 27,159 (1974); S. A. Adelman and D. R. Herschbach, ibid. 33, 793 (1977).

${ }^{16}$ M. B. Faist and R. D. Levine, J. Chem. Phys. 64, 2953 (1976)

${ }^{17}$ H. J. Foth, J. C. Polanyi, and H. H. Telle, J. Phys. Chem. 86, 5027 (1982); H.-J. Foth, H. R. Mayne, R. A. Poirer, J. C. Polanyi, and H. H. Telle, in Photochemistry and Photobiology, edited by A. H. Zewail (Harwood Academic, Chur, Switzerland, 1983), Vol. 1, p. 229.

${ }^{18}$ R. Bersohn, in Alkali Halide Vapors, edited by P. Davidovits and D. L. McFadden (Academic Press, New York, 1979) and references therein.

${ }^{19}$ (a) N. J. A. van Veen, M. S. de Vries, J. D. Sokol, T. Baller, and A. E. de Vries, Chem. Phys. 56, 81 (1981); (b) N. J. A. van Veen, Dissertation, University of Amsterdam, The Netherlands (1980); quoted in (a).

${ }^{20}$ W. R. Anderson, B. M. Wilson, and T. L. Rose, Chem. Phys. Lett. 48, 284 (1977).

${ }^{21}$ S. H. Schaefer, D. Bender, and E. Tiemann, Chem. Phys. 89, 65 (1984); Chem. Phys. Lett. 92, 273 (1982).

${ }^{22}$ H. Beutler and H. Levi, Elektrochem. Angew. Phys. Chem. A 8, 1 (1931); Vorber. Vortr. Hauptversamml. Dtsch. Bunsen-Ges. (1932); Z. Phys. Chem. Abt. B 24, 263 (1934).

${ }^{23}$ R. S. Berry, J. Chem. Phys. 27, 1288 (1957).

${ }^{24}$ R. A. Berg and G. W. Skewes, J. Chem. Phys. 51, 5430 (1969).

${ }^{25}$ (a) K. J. Kaufmann, J. R. Lawter, and J. L. Kinsey, J. Chem. Phys. 60, 4016 (1973); (b) K. J. Kaufmann, J. L. Kinsey, H. B. Palmer, and A. Tewarson, ibid. 60, 4023 (1973); 61, 1865 (1974).

${ }^{26}$ G. A. L. Delvigne and J. Los, Physica 67, 166 (1973)

${ }^{27}$ A. S. Ragone, D. H. Levy, and R. S. Berry, J. Chem. Phys. 77, 3784 (1982).

${ }^{28}$ S. H. Schaefer, D. Bender, and E. Tiemann, J. Chem. Phys. 78, 6341 (1983); R. S. Berry and D. H. Levy, ibid. 78, 6342 (1983)

${ }^{29}$ M. S. Child, in Mol. Spectrosc. (Specialist Periodical Report, The Chemical Society, London, 1974), edited by R. F. Barrow, D. A. Long, and J. J. Millen, Vol. 2, p. 466; in Atom-Molecule Collision Theory, edited by R. B. Bernstein (Plenum, New York, 1979), p. 427.

${ }^{30}$ R. D. Bower, P. Chevrier, P. Das, H. J. Foth, J. C. Polanyi, M. G. Prisant, and J. P. Viscot, J. Chem. Phys. 89, 4478 (1988).

${ }^{31}$ V. Engel, H. Metiu, R. Almeida, R. A. Marcus, and A. H. Zewail, Chem. Phys. Lett. 152, 1 (1988); V. Engel and H. Metiu, J. Chem. Phys. 90, 6116 (1989).

${ }^{32}$ S. E. Choi and J. C. Light, J. Chem. Phys. 90,2593 (1989).

${ }^{33}$ R. A. Marcus, Chem. Phys. Lett. 152, 8 (1988)

${ }^{34}$ S. H. Lin and B. Fain, Chem. Phys. Lett. 155, 216 (1989).

${ }^{35}$ R. L. Fork, C. V. Shank, C. Hirlimann, R. Yen, and W. J. Tomlinson, Opt. Lett. 8, 1 (1983).

${ }^{36}$ C. R. Vidal and J. Cooper, J. Appl. Phys. 40, 3370 ( 1969); C. R. Vidal and M. M. Hessel ibid. 43, 2776 (1972).

${ }^{37}$ A. Gaupp, P. Kuske, and H. J. Andrä, Phys. Rev. A 26, 3351 (1982).

${ }^{38}$ Successive scans were taken with first the pump light only, then the probe only. As expected, the background was found in each case to be independent of the actuator position.

${ }^{39}$ L. E. Brus, J. Chem. Phys. 52, 1716 ( 1976).

${ }^{40}$ M. Dantus, R. Bowman, S. Baskin, and A. H. Zewail, Chem. Phys. Lett. 159, 406 (1989); R. Bowman, M. Dantus, and A. H. Zewail, ibid. 161, 297 (1989).

${ }^{41}$ A. H. Zewail, J. Chem. Soc., Faraday Trans. 2 85, 1221 (1989).

${ }^{42}$ R. Bersohn and A. H. Zewail, Ber. Bunsenges. Phys. Chem. 92, 373 (1988).

${ }^{43}$ Figure 11 demonstrates that the off-resonance signal becomes significant at the red and blue wavelengths of $586 \mathrm{~nm}$ and below. (Tuning to the red was not possible since the narrow band interference filter was centered at $589 \mathrm{~nm}$.) In comparison to the broad bandwidth data, the temporal resolution for these scans is obviously less because of the reduction in bandwidth. However, because the continuum pulses are not transform limited, 
the reduction in resolution is not a factor of 10 . Although autocorrelations of the narrow band pulses were not obtained, their temporal widths were estimated from the width of the first peak in the off-resonance data and were found to be approximately $600-700 \mathrm{fs}$.

${ }^{44}$ It should be mentioned that in the $\lambda_{2}^{*}$ probing experiments, the first peak appears, in some cases, somewhat less in amplitude than the second peak. We are currently examining these control conditions (such as polarization, scattering, etc.) to obtain the actual contributions of the $0^{+}$and 1 state reaction channels.

${ }^{45}$ P. Davidovits and D. C. Broadhead, J. Chem. Phys. 46, 2968 (1967).

${ }^{46}$ S. O. Williams and D. G. Imre, J. Phys. Chem. 92, 6648 (1988).

${ }^{47}$ Note that we have slightly modified the terminology used in Refs. 16, 19 (a), and 31 (the potential surfaces have been renumbered) in order to retain consistency with the description of FTS in our other papers.

${ }^{48}$ E. S. Rittner, J. Chem. Phys. 19, 1030 (1951).

${ }^{49}$ See Ref. 30 and references therein.

${ }^{50}$ H. G. Hanson, J. Chem. Phys. 23, 1391 (1955).

${ }^{51}$ J. R. Barker and R. E. Weston, Jr., J. Chem. Phys. 65, 1427 (1976)

${ }^{52}$ W. H. Press, B. P. Flannery, S. A. Teukolsky, and W. T. Vetterling, Nu- merical Recipes in C (Cambridge University Press, Cambridge, 1988), p. 569.

${ }^{53}$ J. C. Light, I. P. Hamilton, and J. V. Lill, J. Chem. Phys. 82, 1400 (1985). ${ }^{54}$ See E. J. Heller, in Potential Energy Surfaces and Dynamics Calculations, edited by D. Truhlar (Plenum, New York, 1981) and references therein.

${ }^{55}$ While a full pump wavelength dependence for $\mathrm{NaI}$ has been performed, the studies for $\mathrm{NaBr}$ have been limited to a pump wavelength of $\sim 310$ $\mathrm{nm}$. Extensive studies of $\mathrm{NaBr}$ were not possible with the current apparatus since the sample quickly reacted with the quartz cell. According to the work of van Veen et al. (Ref. 19) excitation of the bromide at this wavelength predominately accesses the $\Omega=1$ state (diabatic). Efforts are underway to tune into the wavelength region where the $0^{+}$state absorption is more significant.

${ }^{56}$ The relationship is a consequence of the classical action angle variable $v=\partial H / \partial J$ (where $J$ is the action variable and $J=h n$ ); see, e.g., H. Goldstein, Classical Mechanics, 1st ed. (Addison-Wesley, Reading, MA, 1950), p. 298.

${ }^{57}$ S. Y. Lee, W. T. Pollard, and R. A. Mathies, J. Chem. Phys. 90, 6146 (1989). 